
\title{
R Research S Surare \\ Deployment of Hub-Spoke Tethered Satellite Formation with Adaptive Sliding Mode Tension Control
}

\section{Chenguang Liu}

Northwestern Polytechnical University

\section{Wei Wang}

Northwestern Polytechnical University

\section{Junjie Kang}

York University - Keele Campus: York University

Zheng H. Zhu ( $\sim$ gzhu@yorku.ca )

York University - Keele Campus: York University https://orcid.org/0000-0002-0149-0473

\section{Research Article}

Keywords: Tethered satellite formation, Hub-Spoke, Tether deployment, Sliding mode control, Adaptive control, Multibody dynamics

Posted Date: July 6th, 2021

DOl: https://doi.org/10.21203/rs.3.rs-629893/v1

License: (9) This work is licensed under a Creative Commons Attribution 4.0 International License. Read Full License 


\title{
Deployment of Hub-Spoke Tethered Satellite Formation with Adaptive Sliding Mode Tension Control
}

\author{
Chenguang Liu ${ }^{\text {a, b }}$, Wei Wang a , Junjie Kang ${ }^{\text {b }}$, Zheng H. Zhu ${ }^{\text {b* }}$ \\ ${ }^{a}$ School of Automation, Northwestern Polytechnical University, Xi'an, Shaanxi 710072, China \\ ${ }^{\mathrm{b}}$ Department of Mechanical Engineering, York University, Toronto, Ontario M3J 1P3, Canada
}

\begin{abstract}
This paper studies the deployment control of a spinning hub-spoke tethered satellite formation, which is a challenging issue due to the strong nonlinear coupling between the hub and sub-satellites, and the underactuated nature of the system if no thrust is used for control. The mathematical model of the formation system is established based on the assumption of rigid body of the hub, inextensible tether, and lumped masses of the sub-satellites. Two novel formation deployment controllers are proposed based on tension control and hybrid tension-thrust control strategies, where underactuated sliding mode control and nonsingular terminal sliding mode control method are used, respectively. The adaptive control theory is adopted to estimate the unknown upper bound of the gravitational perturbation caused by the rotation of the system around the hub. It can be proven by the Lyapunov theory that the close-loop systems have bounded and asymptotic stability under these two deployment controllers, respectively. Finally, numerical simulations are conducted to validate the effectiveness and robustness of the proposed controllers.
\end{abstract}

Keywords: Tethered satellite formation; Hub-Spoke; Tether deployment; Sliding mode control;

\footnotetext{
* Corresponding author. Tel: +1 4167362100 x 77729, Email address: gzhu@ yorku.ca
} 
Adaptive control; Multibody dynamics.

\section{Introduction}

Satellite formation flying is a promising technology that can increase the redundancy and robustness of space missions by using multiple small satellites, thereby replacing a large single satellite in the future [1]. However, in order to keep the formation, regular orbit corrections are needed for satellites in the constellation to eliminate orbital drift, which accelerates fuel consumption and reduces the on-orbit life of satellites.

As a new type of satellite formation flying, tethered satellite formation (TSF) has generated a lot of interest in recent years. The satellites in TSF are connected by tethers to keep a specific constellation in orbit [2], leading high accuracy of formation with much less fuel consumption. Several TSF constellations are proposed in the literatures based on mission requirements, such as straight chain [3], hub-spoke [4], triangular ring [5], and double pyramid [6], just to name a few. Among them, the hub-spoke tethered satellite formation (HS-TSF) has attracted extensive attention, especially in the solar wind sail [7] and multi-point measurement missions [8]. Zhao and Cai [9] studied the dynamics of hub-spoke constellation in halo orbits and showed that the HSTSF performs better than free formation in terms of long-term stability of constellation. Avanzini and Fedi $[10,11]$ discussed the spin stability of hub-spoke constellation in Earth orbits by considering the effects of orbital eccentricity and elasticity of tethers. They found that the constellation can keep rotational stability in the orbit plane with a larger rotation speed to reduce the effect of gravitational perturbations and external disturbances. However, the strong nonlinearity and state coupling of HS-TSF make tether deployment very challengeable, which is critical for any space tether missions. The merit of rotational stability of HS-TSF by the centrifugal effect makes rotation deployment widely adopted. One feasible method is the deployment strategy 
of Yo-Yo de-spin systems, where the tethers connecting two payloads are pre-wound around the central body [12]. However, it is difficult to conveniently deploy multiple payloads due to the complicated design of the Yo-Yo mechanism containing multiple tethers. Another popular deployment strategy is the radial deployment $[13,14]$. The deployer of each payload is independent and is placed in the central body together with tethers. This design can be easily extended to multiple payloads, which is more suitable for HS-TSF systems.

Significant research has been undertaken to study the motion of TSF systems located around the second Lagrangian point, because the gravitational and centripetal accelerations at this point are balanced, which is considered to be a fairly suitable condition for a spinning TSF. Wong et al. [15] proposed a linear length law and exponential length law to control the tether deployment of HS-TSF at the L2 point. Zhao et al. [16] employed the presented length laws in [15], and studied the stability of the formation during three-dimensional tether deployment/retrieval. It was found that under the constant tether law the libration motions of the studied linear three-body TSF system are stable during deployment, while under the exponential tether law the out-plane libration departs from the desired position, which means it is difficult for the system to maintain its initial configuration under the latter control law. The mentioned velocity control strategy for the deployment of tethers is characterized by simplicity in terms of the control form. However, it is not sufficient to ensure the stability of libration motions, more control effort is required to guarantee a successful deployment. One feasible approach is to employ thrusters in the deployment control strategy. In [17], apart from the constant tether length law, thrust forces, acting on the subsatellites of a TSF system at the L2 point, are applied to keep the flexible tethers taut and enhance the control of libration motions during tether deployment.

Compared with the TSF system at the Lagrangian points or Halo orbits, when the formation 
system spins on the Earth orbit, it will always be affected by the gravitational perturbations. The dynamics and control problems of such systems have also been studied for a wild range of cases. Chong and Misra [18] found that the hub-spoke constellation can spin stably in both the orbital plane and the plane normal to the orbit. Zhai et al. [19] presented different deployment strategies for a HS-TSF system in the Earth orbit plane depending on if there is active thrust to compensate perturbations or not, such as deployment with constant spinning rate and libration angle. To get better deployment results, the optimal control method was widely used to control the tether deployment of HS-TSF with specific criteria. Without the consideration of gravity gradient and with the constant deployment velocity, the criterion of optimal tether deployment control, solved by the Gauss pseudospectral method, was to minimize the torque acting on the central satellite of the two-body HS-TSF [20]. With the consideration of the gravity perturbation in the Earth orbit plane, the optimal deployment control based on the Pontryagin's minimum principle was to control tether tension and thrusts to obtain the deployment profiles with minimized power consumption [21]. The deployment problem based on optimal control method treats the desired deployment state as one of the constraints, so the libration angles during deployment could strictly converge to zero. Although effective, these optimal controllers are computationally heavy and are usually done off-line. This makes the tether deployment of HS-TSF an open-loop control problem, which cannot response to environment disturbances effectively. Particularly, the situation is further complicated by the periodic gravitational perturbances in the tether deployment of HS-TSF on the Earth orbit.

To address the above challenges, close-loop deployment controllers should be developed to guarantee the global stability of the tethered formation in the presence of perturbances. Sliding mode control (SMC) is regarded as one of the widely used methods due to its strong robustness, and has been successfully applied to the deployment of two-body tethered system. An adaptive 
sliding mode controller was proposed in [22] to deploy an electro-dynamic tether with the consideration of partial unknown parameters. For a more extreme situation, when the nonlinear dynamic model has uncertainty and there exist external disturbances, the terminal sliding mode control (TSMC) approach combined with neural network was employed to deploy a dual-body tethered system [23]. In addition, in order to achieve better dynamic responses, fractional order SMC method, characterized by its favorable historical memory effect, was applied to control the process of tether deployment $[24,25]$. Although the SMC method-based deployment control has been extensively studied in dual-body tethered systems, few studies have been presented on its application in multi-body TSF systems. An adaptive hierarchical SMC was adopted to stabilize the attitude motions of a rotational multi-satellite inline system [26]. Hallaj et al. [27] proposed a robust SMC controller for the reconfiguration and keeping stage of an electromagnetic TSF system.

The deployment control problem of a spinning HS-TSF system within the orbital plane is studied in the current work. The system is assumed to consist of a central satellite modeled as a rigid body and sub-satellites modeled as point masses. The main novelty of this paper is to propose two robust deployment controllers to ensure that the system has stronger anti-interference ability. First, a pure tether tension control strategy based on adaptive underactuated SMC is presented to make full use of the structural characteristics of the considered formation system, thereby leading to lower fuel consumption. Next, a hybrid tension-thrust control strategy based on adaptive TSMC is proposed to reduce the risk of tether entanglement during deployment. The TSMC method adopted in the hybrid controller design is expected to achieve rapid convergence of the control system. Besides, the adaptive laws for these two deployment controllers can estimate the upper bounds of periodic gravitational perturbations in system.

\section{Mathematical Modeling}


The multi-tether system under consideration, as shown in Fig.1, consists of a central satellite and $n$ sub-satellites, individually connected to the central satellite by $n$ tethers. It is assumed that all the tethers are rigid and of negligible mass. The central satellite is treated as a symmetrical rigid body, while the sub-satellites are simplified as a set of point masses. Moreover, the junction points of the tethers are evenly distributed around the central satellite.

Consider the system is rotating in a Keplerian circular orbit. $O-X Y Z$ denotes the Earth-centered inertial frame, in which the origin $O$ is located at the center of the Earth, the $O X$ axis points to the vernal equinox, the $O Z$ axis aligns with the rotation axis of the Earth, and the $O Y$ axis completes the right-hand triad. The unit direction vectors of the $O X, O Y$ and $O Z$ axis are defined by $i, j$ and $\boldsymbol{k}$, respectively. Thus, the orbital angular velocity of the system has the form: $\boldsymbol{\Omega}=\boldsymbol{\Omega} \boldsymbol{k}$.

The motion of the multi-tether system is described with respect to two rotating coordinate systems: the orbital coordinate system $C-x y z$, and the body-fixed coordinate system $C-x_{o} y_{o} z_{o}$. The origin of the two coordinate systems is at the center of mass (CM) of the system. The $C x$ axis is along the direction of the orbital radius vector $\boldsymbol{R}$, the $C y$ axis points to the direction of the orbital motion, and the axis $C z$ is formed by the right-hand rule. Define $r_{i}$ as the radius vector of the $i^{\text {th }}$ junction point, here, $i=1,2, . ., n$. The angle between $r_{i}$ and the $C x$ axis is denoted by $\theta_{i}$, which represents the rotation of the central satellite around its CM. The body-fixed coordinate system $C-x_{0} y_{0} z_{0}$ is used to describe the motion of the tethers. The $C z_{0}$ axis coincides with the $C z$ axis, the $C x_{0}$ axis is along the direction of the radius vector $\boldsymbol{r}_{i}$, and the $C y_{0}$ axis completes the right-hand triad. The length of the $i^{\text {th }}$ tether is represented by $l_{i}$, and the angle $\alpha_{i}$ defines the orientation of the $i^{t h}$ tether with respect to the $C x_{0}$ axis. Besides, it can be seen that the coordinate system $C-x_{0} y_{0} z_{0}$ can be obtained by rotating $C$-xyz around its $C z$ axis by angle $\theta_{i}$. 


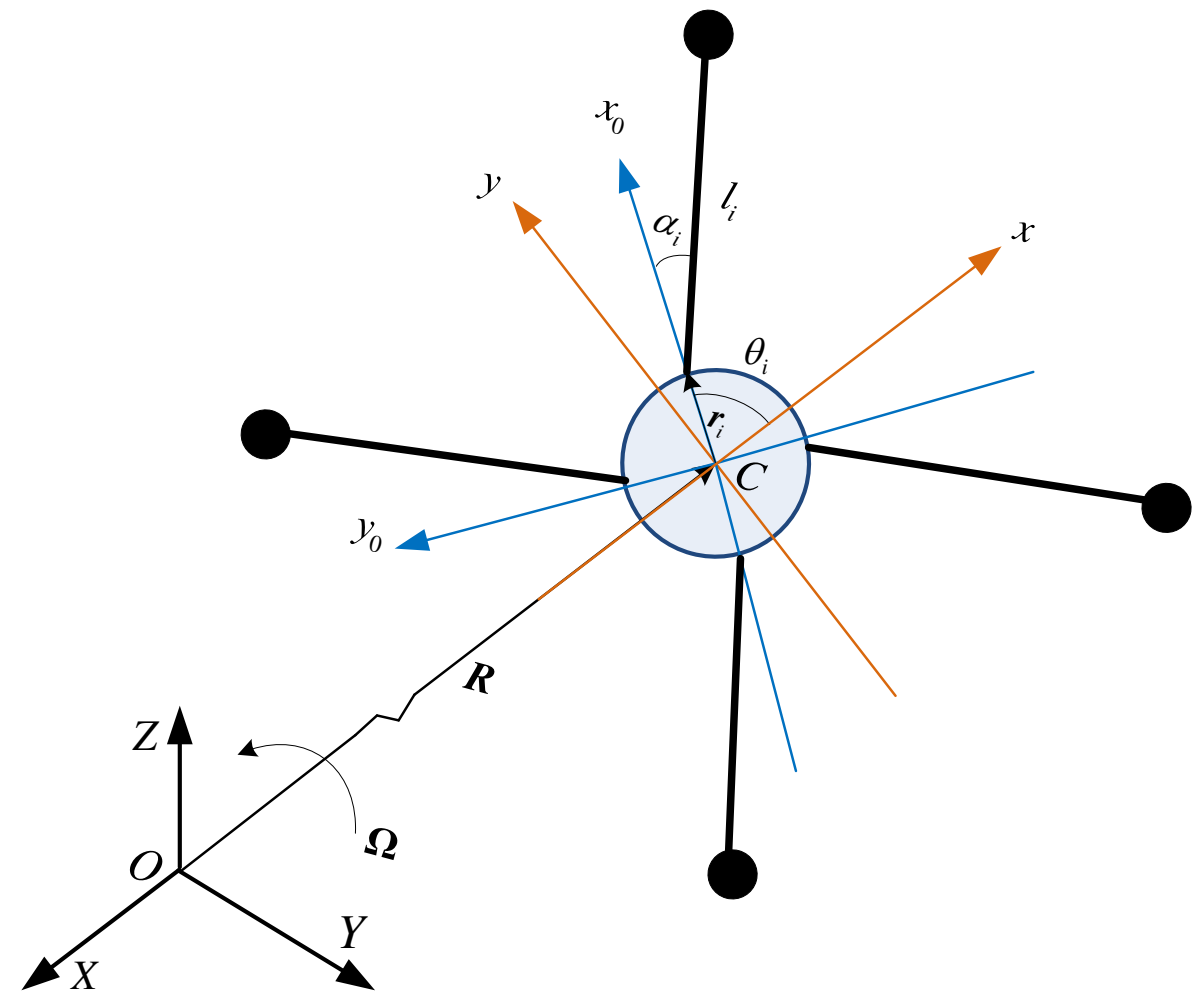

Fig. 1. The schematic diagram of the HS-TSF.

The position vector of the $i$-th external satellite relative to the $\mathrm{CM}$ of the central satellite can be written as:

$$
\boldsymbol{p}_{\boldsymbol{i}}=\left[r \cos \theta_{i}+l_{i} \cos \left(\theta_{i}-\alpha_{i}\right)\right] \boldsymbol{i}_{\boldsymbol{c}}+\left[r \sin \theta_{i}+l_{i} \sin \left(\theta_{i}-\alpha_{i}\right)\right] \boldsymbol{j}_{\boldsymbol{c}}
$$

where $\boldsymbol{i}_{\boldsymbol{c}}$ and $\boldsymbol{j}_{\boldsymbol{c}}$ are the unit vectors of the $C x$ and $C y$ axes, and $r=\left\|\boldsymbol{r}_{\boldsymbol{i}}\right\|$ because of the symmetry property of the central satellite.

Then, the velocity vector of the $i$-th external satellite with respect to the central satellite can be obtained by

$$
v_{i}=p_{l}^{\ell}+\Omega \times p_{i}
$$

Thus, the total kinetic energy of the system can be written as [18]: 


$$
T=\frac{1}{2} \sum_{i=1}^{n} m_{i}\left(v_{c}+v_{i}\right) \cdot\left(v_{c}+v_{i}\right)+\frac{1}{2} m_{c} v_{c}^{2}+\frac{1}{2} J(\omega+\Omega)^{2}
$$

where $m_{c}$ is the mass of the central satellite, $m_{i}$ is the mass of the $i$-th sub-satellite, $J$ represents the moment of inertial of the central satellite, $\boldsymbol{v}_{\boldsymbol{c}}$ is the orbital velocity of CM of the central satellite $\boldsymbol{v}_{\boldsymbol{c}}=\boldsymbol{\Omega} \times \boldsymbol{R}, \quad \omega=\bigotimes_{i}^{\&}$ represents the spin angular velocity of the central satellite.

The total potential energy of the system can be expressed as:

$$
U=-\mu_{e}\left(\frac{m_{c}}{|\boldsymbol{R}|}+\sum_{i=1}^{n} \frac{m_{i}}{\left|\boldsymbol{R}+\boldsymbol{p}_{i}\right|}\right)
$$

where $\mu_{e}$ is the geocentric gravitational constant.

The equations that govern the rotation motion of the central satellite, and the deployment of tethers are derived by using the Lagrangian method:

$$
\frac{d}{d t}\left(\frac{\partial T}{\partial \&_{J}}\right)-\frac{\partial T}{\partial q_{j}}+\frac{\partial U}{\partial q_{j}}=Q_{q_{j}}
$$

where $q_{j}$ are the generalized coordinates, and $Q_{q_{j}}$ are the corresponding generalized forces. For the $i$-th tether, the generalized coordinates are selected as the spinning angle of the central body $\theta_{i}$, the length $l_{i}$ and the libration angle $\alpha_{i}$. The equations of motion can be expressed as follows:

$$
\begin{aligned}
& {\left[J+\sum_{i=1}^{n} m_{i}\left(r^{2}+l_{i}^{2}+2 l_{i} r \cos \alpha_{i}\right)\right] \sum_{i}^{n} \sum_{i=1}^{n} m_{i}\left(l_{i}^{2}+l_{i} r \cos \alpha_{i}\right)-\sum_{i=1}^{n} m_{i} r t_{i} \sin \alpha_{i}}
\end{aligned}
$$

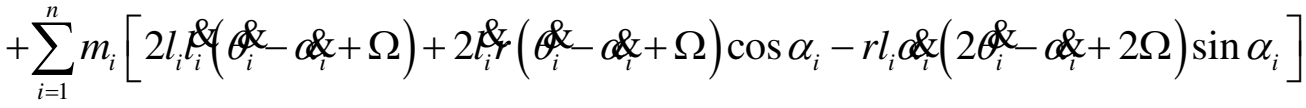

$$
\begin{aligned}
& +3 \Omega^{2} \sum_{i=1}^{n} m_{i}\left[\cos \theta_{i} \sin \theta_{i} r^{2}+l_{i}^{2} \cos \left(\theta_{i}-\alpha_{i}\right) \sin \left(\theta_{i}-\alpha_{i}\right)+r l_{i} \sin \left(2 \theta_{i}-\alpha_{i}\right)\right]=Q_{\theta_{i}}
\end{aligned}
$$




$$
\begin{aligned}
& \&_{l}-\left(1+\frac{r}{l_{i}} \cos \alpha_{i}\right) \oiint_{i}^{\&} 2 \frac{l_{i}}{l_{i}}\left(\beta_{i}^{\&}-\alpha_{i}+\Omega\right)+\frac{r}{l_{i}}\left(\phi_{i}+\Omega\right)^{2} \sin \alpha_{i}- \\
& \Omega^{2}\left[\frac{r}{l_{i}} \sin \alpha_{i}+3 \cos \left(\theta_{i}-\alpha_{i}\right) \sin \left(\theta_{i}-\alpha_{i}\right)+3 \frac{r}{l_{i}} \cos \theta_{i} \sin \left(\theta_{i}-\alpha_{i}\right)\right]=\frac{Q_{\alpha_{i}}}{m_{i} l_{i}^{2}}
\end{aligned}
$$

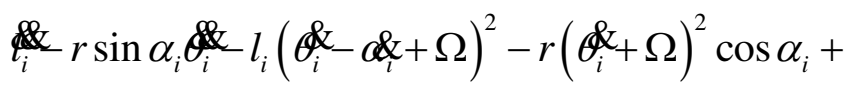

$$
\begin{aligned}
& \Omega^{2}\left(l_{i}+r \cos \alpha_{i}-3 l_{i} \cos ^{2}\left(\theta_{i}-\alpha_{i}\right)-3 r \cos \theta_{i} \cos \left(\theta_{i}-\alpha_{i}\right)\right)=\frac{Q_{l_{i}}}{m_{i}}
\end{aligned}
$$

where $Q_{\theta}, Q_{l_{i}}, Q_{\alpha_{i}}$ are the generalized forces.

It has been common practice in dynamic analysis and control-system design to employ the simplified mathematical model for multi-tether system, in which the central body is assumed to be a mass point [16]. However, such a model is insufficient to demonstrate the coupling dynamics of the central body and the tethers, especially in the deployment phase of a spinning tethered constellation. This is one of the main concerns of the current work, and that's why the central satellite considered here is modeled as a rigid body. But in actual fact, overwhelming complexities exist when it comes to the dynamics behaviors of the system. On the other hand, the attitude motion of the central satellite is often expected to be stable in orbit, and it is promising to reach such a goal by employing proper active control [21]. That's where the approximation condition that the central body spins constantly to simplify the dynamic model and make sense for control design during tether deployment come in. In view of all the considerations above, we assume that $\omega_{c}=\bigotimes_{i}=$ const . And, the equations governing the motion of tethers during deployment can be simplified to some extent and studied separately.

As the non-dimensional equations are more convenient, define the non-dimensional time $\tau=\Omega t$. In order to avoid ambiguity, it is important to note that in the rest of the paper all the derivatives in equations represents derivatives with respect to the non-dimensional time $\tau$. Thus, 
the equations of tether length and librations can be rewritten as follows:

$$
\begin{aligned}
& -2 \frac{l_{i}}{l_{i}}(N-\alpha \&+1)+\frac{r}{l_{i}} \sin \alpha_{i}\left(N^{2}+2 N\right)+g_{\alpha_{i}}=\frac{Q_{\alpha_{i}}}{m_{i} \Omega^{2} l_{i}^{2}} \\
& l_{i}\left[\left(N-\alpha_{l}+1\right)^{2}-1\right]-r \cos \alpha_{i}\left(N^{2}+2 N\right)+g_{l_{i}}=\frac{Q_{l_{i}}}{m_{i} \Omega^{2}}
\end{aligned}
$$

with $N=\omega_{c} / \Omega$ and

$$
\begin{aligned}
& g_{\alpha_{i}}=-3 \cos \left(\theta_{i}-\alpha_{i}\right) \sin \left(\theta_{i}-\alpha_{i}\right)-3 \frac{r}{l_{i}} \cos \theta_{i} \sin \left(\theta_{i}-\alpha_{i}\right), \\
& g_{l_{i}}=-3 l_{i} \cos ^{2}\left(\theta_{i}-\alpha_{i}\right)-3 r \cos \left(\theta_{i}-\alpha_{i}\right) \cos \theta_{i}
\end{aligned}
$$

where $g_{\alpha_{i}}$ and $g_{l_{i}}$ are the periodic gravitational perturbation terms caused by the rotation of the central body.

The generalized forces $Q_{l_{i}}$ and $Q_{\alpha_{i}}$ in Eq. (7) are evaluated using the principle of virtual work. For each sub-satellite, the tether tension $\boldsymbol{T}_{i}$, which is along the tether, and the thrust force $\boldsymbol{F}_{i}$, which is perpendicular to the tether, are proposed as the nonconservative control forces, acting on the sub-satellite. Then, the generalized forces may be obtained as $Q_{l_{i}}=-T_{i}$ and $Q_{\alpha_{i}}=-F_{i} l_{i}$.

\section{Deployment strategies and controller design}

The major goal of the current work is to demonstrate effective control for the deployment process of the HS-TSF using tether tension and thrust force. In this section, first of all, the deployment control problem is described, and then, two controllers are derived using sliding mode control technique.

\subsection{Control Problem Description}

The deployment problem for the considered multi-body tethered system is to deploy the tethers fully to form and maintain the desired configuration. After the tethers are deployed to full 
length at the final time $t_{\mathrm{eq}}$, one has $l_{i}\left(t_{\mathrm{eq}}\right)=l_{\mathrm{eq}_{i}}, t_{i}\left(t_{\mathrm{eq}}\right)=l_{i}\left(t_{\mathrm{eq}}\right)=0$. Furthermore, the equation governing the tether libration motion reduces to:

$$
\frac{r}{l_{\mathrm{eq}_{i}}}\left(N^{2}+2 N\right) \sin \alpha_{i}+g_{\alpha_{i}}=-\frac{F_{i}}{m_{i} \Omega^{2} l_{\mathrm{eq}_{i}}}
$$

where $g_{\alpha_{i}}=-3 \cos \left(\theta_{i}-\alpha_{i}\right) \sin \left(\theta_{i}-\alpha_{i}\right)-3 \frac{r}{l_{\mathrm{eq}_{i}}} \cos \theta_{i} \sin \left(\theta_{i}-\alpha_{i}\right)$.

As shown in Eq. (8), the libration motions for the post-deployment system are always subjected to the perturbation terms $g_{\alpha_{i}}$ generated by the rotation of the system. Since the perturbations can be compensated with the thrust force $F_{i}$, which are provided by the thrusters fixed on the sub-satellites, Eq. (8) can be equivalent as

$$
\frac{r}{l}+\frac{r}{l_{\mathrm{eq}_{i}}}\left(N^{2}+2 N\right) \sin \alpha_{i}=0
$$

which becomes a nonlinear but autonomous equation. The phase space of the equivalent system has two dimensions: $\alpha_{i}$ and $\alpha_{i}$. Besides, as can be noticed, the equation of this system is similar to that of a simple pendulum and has the same positions of equilibria: with the centers at $\pm 2 k \pi$ and saddle points at $\pm(2 k+1) \pi$. Considering the fact that the libration angle is always restricted to the range $\left[\begin{array}{ll}-\pi / 2 & \pi / 2\end{array}\right]$ in order to prevent tether twining during the deployment process, $\alpha_{i}=0, \alpha_{i}^{\&}=0$ becomes the only stable equilibrium state for the libration motion of each tether.

The phase portrait for the tether libration is shown in Fig. 2, when the parameters are chosen as $N=100, l_{\mathrm{eq}_{i}}=100 m, r=1 \mathrm{~m}$ and $F_{i}=0 N$. It's worth pointing out that the phase portrait is actually obtained from Eq. (8) with the perturbation terms taken into consideration. Nevertheless, it can be found that phase trajectories match with the results of the analytical analysis above. That's 
because the magnitude of the periodic perturbation terms is small [21], so it's reasonable to make an approximation by simply ignoring the perturbations in preliminary analytical analysis or regard them as bounded disturbances in the following controller design.

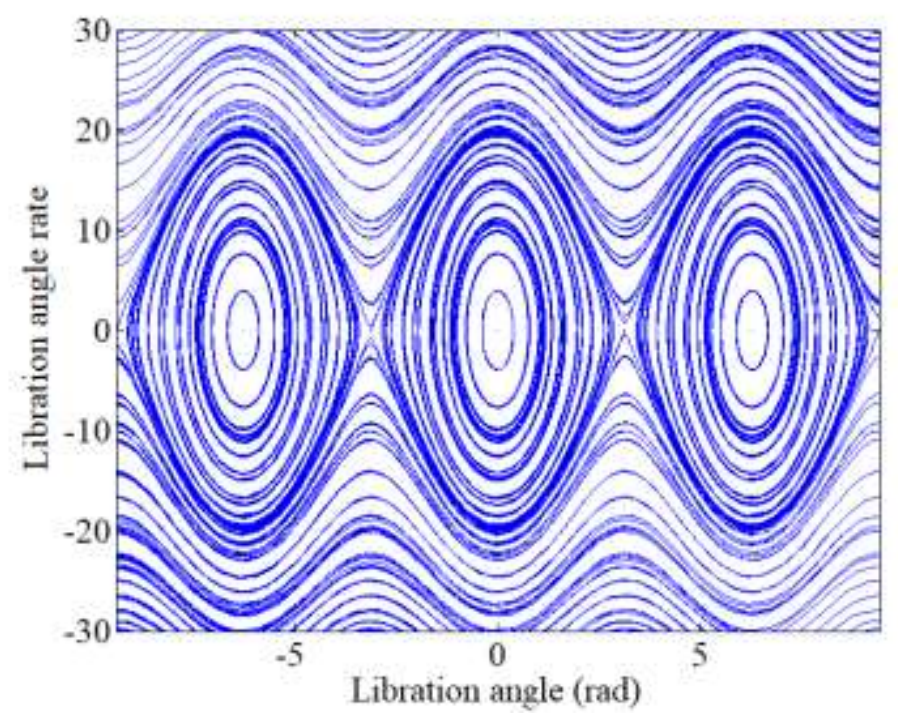

Fig. 2. Phase diagram of the libration angle of tether.

Above all, the desired system states at the final time of the deployment are $l_{i}\left(t_{\mathrm{eq}}\right)=l_{\mathrm{eq}_{i}}$, $l_{i}^{\&}\left(t_{\mathrm{eq}}\right)=0, \alpha_{i}\left(t_{\mathrm{eq}}\right)=0$ and $\alpha_{i}^{\&}\left(t_{\mathrm{eq}}\right)=0$. The other thing needed to note here is the strong coupling between the tether deployment and libration motions before the deployment reaches its final state. From the analytical aspect, the coupling can be explained by the Coriolis term $-2 \frac{l_{i}^{\&}}{l_{i}}\left(N-a_{i}^{\&}+1\right)$, as shown in Eq. (7-1), and it is obvious that the coupling becomes stronger with the deployment velocity increasing. Fig. 3 shows several phase portraits for the libration motions at different deployment velocities ( $\left.\mathbb{R}_{i}^{\&}=1,10,50,100\right)$. As the deployment velocity increases, there are much more crossover movements between the centers, which means the tether deployment will affect the libration motion and may even cause tether twining around the central satellite. Therefore, a proper deployment control law is required to ensure the stability of the process. 

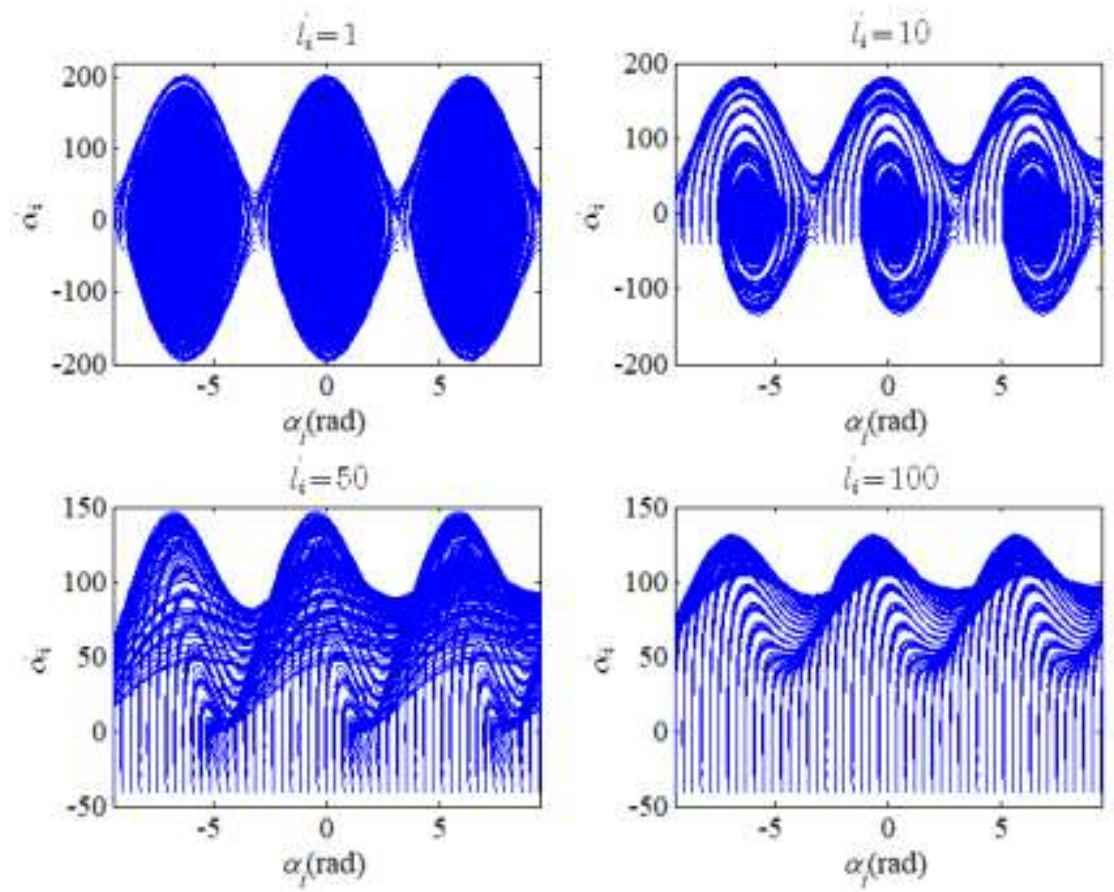

Fig. 3. Phase diagrams of the libration angle in the different cases.

\subsection{Controller Design}

Notice that the dynamic equations of each sub-satellite have the same form, without loss of generality, the subscript $i$ on any symbol is ignored in the following sections.

Define the system state vector as $\boldsymbol{x}=\left[x_{1}, x_{2}, x_{3}, x_{4}\right]^{\mathrm{T}}=\left[\alpha, \alpha \& l, l^{\mathrm{T}}\right]^{\mathrm{T}}$. After full deployment of tethers, the formation system is expected to achieve the final state: $\boldsymbol{x}_{\mathrm{eq}}=\left[0,0, l_{\mathrm{eq}}, 0\right]^{\mathrm{T}}$. In order to make it convenient for controller design, we introduce the transformation $L=l-l_{e q}$, so that all the system states will converge to 0 at the final time of the deployment. In this way, the new system state vector becomes $\boldsymbol{x}=\left[x_{1}, x_{2}, x_{3}, x_{4}\right]^{\mathrm{T}}=[\alpha, \alpha, L, \&]^{\mathrm{T}}$. And the control task can be described as making the system move from its initial states $\left[\alpha_{0}, \alpha \&, L_{0}, \&_{0}^{\&}\right]^{\mathrm{T}}$ to the origin $[0,0,0,0]^{\mathrm{T}}$.

Then, the dynamic model of the formation system can be expressed in a reduced-order form: 


$$
\left\{\begin{array}{l}
\not \&=x_{2} \\
\&=f_{\alpha}+g_{\alpha}+u_{\alpha} \\
\&=x_{4} \\
\&=f_{l}+g_{l}+u_{l}
\end{array}\right.
$$

where the functions $f_{\alpha}$ and $f_{l}$ are:

$$
\begin{aligned}
& f_{\alpha}=2 \frac{x_{4}}{x_{3}+l_{e q}}\left(N+1-x_{2}\right)-\frac{r}{x_{3}+l_{e q}} \sin x_{1}\left(N^{2}+2 N\right), \\
& f_{l}=\left(x_{3}+l_{e q}\right)\left[\left(N+1-x_{2}\right)^{2}-1\right]+r \cos x_{1}\left(N^{2}+2 N\right)
\end{aligned}
$$

the perturbation terms are:

$$
\begin{aligned}
& g_{\alpha}=3 \cos \left(\theta-x_{1}\right) \sin \left(\theta-x_{1}\right)+3 \frac{r}{x_{3}+l_{e q}} \cos \theta \sin \left(\theta-x_{1}\right), \\
& g_{l}=3\left(x_{3}+l_{e q}\right) \cos ^{2}\left(\theta-x_{1}\right)+3 r \cos \theta \cos \left(\theta-x_{1}\right)
\end{aligned}
$$

and the control inputs are: $u_{\alpha}=\frac{-F}{m \Omega^{2}\left(x_{3}+l_{e q}\right)}$ and $u_{l}=\frac{-T}{m \Omega^{2}}$.

To make sure that the tethers can be deployed successfully, the initial value for tether length needs to be greater than 0 , that is $L_{0}+l_{e q}>0$, which guarantees the perturbation terms are always bounded. And the unknown upper bounds are assumed as $\left|g_{\alpha}\right| \leq \delta_{\alpha}$ and $\left|g_{l}\right| \leq \delta_{l}$, where $\delta_{\alpha}$ and $\delta_{l}$ are positive constant numbers. Thus, in the controller design the perturbation terms can be treated as disturbances with upper limits.

\section{(I) Tension Controller}

The advantage of employing tension control is that no fuel consumption is required to control the sub-satellites in the deployment process, except for the tether tension. The presented control problem can be solved using adaptive underactuated sliding mode control method, and the controller with anti-disturbance capacity is proposed to guarantee the stability of the deployment 
process.

Rewrite Eq. (10) as

$$
\left\{\begin{array}{l}
\&=x_{2} \\
\&=\bar{f}_{\alpha}+\bar{g}_{\alpha} \\
\&=x_{4} \\
\&=f_{l}+g_{l}+u_{l}
\end{array}\right.
$$

where $\bar{f}_{\alpha}=f_{\alpha}+\varepsilon x_{3}, \bar{g}_{\alpha}=g_{\alpha}-\varepsilon x_{3}$, and $\varepsilon$ is a positive parameter.

The desired nonlinear sliding mode surface of the proposed control system can be designed as:

$$
s=c_{1} e_{1}+c_{2} e_{2}+e_{3}
$$

where $e_{1}=x_{1}$ and $e_{2}=x_{2}$ denote the errors of the libration angle and libration angle rate, respectively; $c_{1}$ and $c_{2}$ are control parameters, and satisfy $c_{1}>0, c_{2}>0 ; e_{3}=\bar{f}_{\alpha}=f_{\alpha}+\varepsilon x_{3}$ represents the nonlinear term of the sliding mode surface, and this term is introduced to ensure the stability of the motion of tether length, that is, when $e_{3} \rightarrow 0$, one can obtain $x_{3} \rightarrow 0, x_{4} \rightarrow 0$, which means the tethers are deployed to the desired length and the deployment velocity converges to 0 . Correspondingly, the term $-\varepsilon x_{3}$ is added to the previous perturbation term $g_{\alpha}$, and together form the new perturbation term $\bar{g}_{\alpha}$. It is worth noting that the parameter $\varepsilon$ shouldn't be too small, otherwise the accuracy of tether length control cannot be ensured, but on the other, if $\varepsilon$ is too large, then the new perturbation term will be greatly enhanced, which may exceed the anti-interference ability of the tension controller.

Taking derivative of the sliding mode surface in Eq. (12) yields: 


$$
\&=c_{1} x_{2}+c_{2} \bar{f}_{\alpha}+\frac{\partial \bar{f}_{\alpha}}{\partial x_{1}} x_{2}+\frac{\partial \bar{f}_{\alpha}}{\partial x_{2}} \bar{f}_{\alpha}+\frac{\partial \bar{f}_{\alpha}}{\partial x_{3}} x_{4}+\frac{\partial \bar{f}_{\alpha}}{\partial x_{4}}\left(f_{l}+u_{l}\right)+D
$$

where $D=c_{2} \bar{g}_{\alpha}+\frac{\partial \bar{f}_{\alpha}}{\partial x_{2}} \bar{g}_{\alpha}+\frac{\partial \bar{f}_{\alpha}}{\partial x_{4}} g_{l}$ is the sum of the terms related to the perturbations. It can be found from the underactuated formation deployment model, as shown in Eq. (11), that $\frac{\partial \bar{f}_{\alpha}}{\partial x_{2}}=-2 \frac{x_{4}}{x_{3}+l_{\text {eq }}}$ and $\frac{\partial \bar{f}_{\alpha}}{\partial x_{4}}=\frac{2}{x_{3}+l_{\text {eq }}}\left(N+1-x_{2}\right)$ are bounded. In addition, by taking into account the boundness of the perturbations in Eq. (10) together, one can find that $D$ is also bounded $|D| \leq \delta_{D}$, where $\delta_{D}$ denotes the unknown upper boundary.

The underactuated tension controller is proposed as:

$$
u_{l}=-\left(\frac{\partial \bar{f}_{\alpha}}{\partial x_{4}}\right)^{-1}\left(c_{1} x_{2}+c_{2} \bar{f}_{\alpha}+\frac{\partial \bar{f}_{\alpha}}{\partial x_{1}} x_{2}+\frac{\partial \bar{f}_{\alpha}}{\partial x_{2}} \bar{f}_{\alpha}+\frac{\partial \bar{f}_{\alpha}}{\partial x_{3}} x_{4}+\hat{\delta}_{D} \operatorname{sgn}(s)+\eta s\right)-f_{l}
$$

where the parameter $\eta>0 ; \hat{\delta}_{D}$ is the estimated value for $\delta_{D}$, and the error of estimation is defined as $\delta_{D}^{o}=\hat{\delta}_{D}-\delta_{D} ; \operatorname{sgn}(\bullet)$ denotes signum function. The adaptive law for the estimation of the upper boundary $\delta_{D}$ is presented as:

$$
\delta_{D}^{\&}=\frac{1}{\gamma}|s|
$$

where the parameter $\gamma$ determines the speed of the estimation and $\gamma>0$.

Theorem 1: For the underactuated tethered formation system Eq. (11), if the controller is proposed as Eq. (14), meanwhile the adaptive law for the upper boundary of the sum of perturbation terms is designed as Eq. (15), then the HS-TSF can be deployed into a neighborhood of the equilibrium state.

Proof: Firstly, it can be proven that the sliding surface, as shown in Eq. (13), asymptotically 
converges to 0 according to the Lyapunov stability analysis. The details of the proof procedure are shown in the Appendix A.

When the control system reaches the sliding mode surface $s=0$, then $e_{3}=-c_{1} e_{1}-c_{2} e_{2}$. To illustrate the convergence of the system states, a new system is introduced:

$$
\left\{\begin{array}{c}
\&=e_{2} \\
\&=-c_{1} e_{1}-c_{2} e_{2}+\bar{g}_{\alpha}
\end{array}\right.
$$

Define $\boldsymbol{E}=\left[e_{1}, e_{2}\right]^{\mathrm{T}}$, Eq. (16) can be written as:

$$
\boldsymbol{E}=\boldsymbol{A}_{e} \boldsymbol{E}+\boldsymbol{B}_{e}
$$

where

$$
\boldsymbol{A}_{e}=\left[\begin{array}{cc}
0 & 1 \\
-c_{1} & -c_{2}
\end{array}\right], \quad \boldsymbol{B}_{e}=\left[\begin{array}{c}
0 \\
\bar{g}_{\alpha}
\end{array}\right]
$$

Considering the boundness of $g_{\alpha}$, the perturbation term $\bar{g}_{\alpha}$ is also bounded, which can be denoted as $\bar{g}_{\alpha} \leq\left|\bar{g}_{\alpha}\right| \leq \delta_{g \alpha}$, where $\delta_{g \alpha}$ is the upper boundary. Therefore, the matrix $\boldsymbol{B}_{e}$ in Eq. (17) yields the relationship $\left\|\boldsymbol{B}_{e}\right\|_{2} \leq \delta_{g \alpha}$. It can be found that the poles of the matrix $\boldsymbol{A}_{e}$ are $\frac{-c_{2} \pm \sqrt{c_{2}^{2}-4 c_{1}}}{2}$, and there always exist a negative real part in the poles, so $A_{e}$ is a Hurwitz matrix. Then, by introducing a positive definite matrix $\boldsymbol{Q}$, the Lyapunov equation $\boldsymbol{P} \boldsymbol{A}_{e}+\boldsymbol{A}_{e}^{\mathrm{T}} \boldsymbol{P}=-\boldsymbol{Q}$ will have a unique solution $\boldsymbol{P}$, which is also a positive definite matrix. Furthermore, $V_{e}=\boldsymbol{E}^{\mathrm{T}} \boldsymbol{P E}$ can be used as a Lyapunov function, and the derivation of the Lyapunov function is obtained as: 


$$
\begin{aligned}
V_{e}^{\&} & =-\boldsymbol{E}^{\mathrm{T}} \boldsymbol{Q} \boldsymbol{E}+\frac{\partial V_{e}}{\partial \boldsymbol{E}} \boldsymbol{B}_{e} \\
& \leq-\lambda_{\min }(\boldsymbol{Q})\|\boldsymbol{E}\|_{2}^{2}+\left\|\frac{\partial V_{e}}{\partial \boldsymbol{E}}\right\|_{2}\left\|\boldsymbol{B}_{e}\right\|_{2} \\
& \leq-\lambda_{\min }(\boldsymbol{Q})\|\boldsymbol{E}\|_{2}^{2}+2 \delta_{g \alpha} \lambda_{\max }(\boldsymbol{P})\|\boldsymbol{E}\|_{2}
\end{aligned}
$$

Define a constant $0<\psi<1$,

$$
\begin{aligned}
& V_{e}^{\&} \leq-(1-\psi) \lambda_{\min }(\boldsymbol{Q})\|\boldsymbol{E}\|_{2}^{2}-\psi \lambda_{\text {min }}(\boldsymbol{Q})\|\boldsymbol{E}\|_{2}^{2}+2 \delta_{g \alpha} \lambda_{\max }(\boldsymbol{P})\|\boldsymbol{E}\|_{2} \\
& \quad \leq-(1-\psi) \lambda_{\min }(\boldsymbol{Q})\|\boldsymbol{E}\|_{2}^{2}, \quad \forall\|\boldsymbol{E}\|_{2} \geq \frac{\delta_{g \alpha}}{\psi} \frac{2 \lambda_{\max }(\boldsymbol{P})}{\lambda_{\text {min }}(\boldsymbol{Q})}
\end{aligned}
$$

where $\lambda_{\max }(\bullet)$ and $\lambda_{\text {min }}(\bullet)$ denote the largest and smallest eigenvalues of a matrix, respectively.

It can be seen that Eq. (19) guarantees the globally uniformly ultimate boundness of the system shown in Eq. (17). Based on the discussions above, it is reasonable to conclude that the states $e_{1}$ and $e_{2}$ could gradually converge to a neighborhood of zero $o_{1}$. As a result, $e_{3}=\bar{f}_{\alpha}$ also converges to a neighborhood of zero because of the relationship $e_{3}=-c_{1} e_{1}-c_{2} e_{2}$. Moreover, when $e_{1}, e_{2} \rightarrow o_{1}$, that is, $x_{1}, x_{2} \rightarrow o_{1}$, the nonlinear function $\bar{f}_{\alpha}$ in Eq. (11) can be rewritten as:

$$
\begin{aligned}
\bar{f}_{\alpha}\left(o_{1}, o_{1}, x_{3}, x_{4}\right) & =2 \frac{x_{4}}{x_{3}+l_{\text {eq }}}(N+1)+\varepsilon x_{3}-2 \frac{x_{4}}{x_{3}+l_{\text {eq }}} o_{1}-\frac{r\left(N^{2}+2 N\right)}{x_{3}+l_{\text {eq }}} o_{1} \\
& =2 \frac{x_{4}}{x_{3}+l_{\text {eq }}}(N+1)+\varepsilon x_{3}+o_{2}
\end{aligned}
$$

where $o_{2}$ is the neighborhood caused by the nonlinear terms related to $o_{1}$. Thus, the nonlinear part $2 \frac{x_{4}}{x_{3}+l_{\mathrm{eq}}}(N+1)+\varepsilon x_{3}$ in Eq. (20) is also within a neighborhood of zero. Consequently, the states $x_{3}$ and $x_{4}$ could also converge to the neighborhoods. 
In summary, all the states of the formation system will converge to the neighborhoods of zero at the final time of the deployment. In other words, the HS-TSF considered in the current work could be deployed into the neighborhood of the desired equilibrium state under the proposed tension controller. Thus, Theorem 1 is proved.

Remark 1: The ratio $2 \lambda_{\max }(\boldsymbol{P}) / \lambda_{\min }(\boldsymbol{Q})$, as shown in Eq. (19), is expected to be as small as possible in order to obtain a smaller ultimate boundary of the neighborhood of zero $o_{1}$. Actually, this ratio can be minimized by setting $\boldsymbol{Q}$ as a unit matrix [28], then we have $\lambda_{\min }(\boldsymbol{Q})=1$ and $2 \lambda_{\max }(\boldsymbol{P})=1 / \lambda_{\text {left }}\left(-\boldsymbol{A}_{e}\right)$, where $\lambda_{\text {left }}(\bullet)$ denotes the real part of the leftmost eigenvalue of a matrix. Consequently, the ratio $2 \lambda_{\max }(\boldsymbol{P}) / \lambda_{\min }(\boldsymbol{Q})$ will decrease as the eigenvalue $\lambda_{\text {eft }}\left(-\boldsymbol{A}_{e}\right)$ increases, and it is easily found from Eq. (17) that $\lambda_{\text {left }}\left(-\boldsymbol{A}_{e}\right)$ can be suitably assigned by selecting the parameters $c_{1}$ and $c_{2}$.

Remark 2: The pure tension control method provides the possibility to deploy the tethers without consuming fuel on the sub-satellites. The tension controller is designed based on the nonlinear underactuated SMC method [28], besides, the term $\varepsilon x_{3}$ is introduced into the system during the controller design to guarantee the accuracy of the tether length control. Furthermore, considering the inherent periodic perturbations in the dynamic system, an adaptive law is combined with the controller to enhance the anti-disturbance capability of the system.

\section{(II) Hybrid Tension-Thrust Controller}

It is well-known that keeping the libration angle within an allowable range during the tether deployment is hardly guaranteed using pure tension control strategy. In order to reduce the risk of entanglement between tethers and the central satellite, a hybrid tension-thrust control strategy is 
presented in this section, and the controller is designed based on the terminal sliding mode control method.

Considering the system model shown in Eq. (10), define the deployment error signals as:

$$
\boldsymbol{e}=\left[\begin{array}{ll}
e_{\alpha} & e_{l}
\end{array}\right]^{\mathrm{T}}=\left[\begin{array}{ll}
x_{1} & x_{3}
\end{array}\right]^{\mathrm{T}}
$$

Then, the nonsingular terminal sliding surfaces are given as follows [29]:

$$
s_{j}=e_{j}+k_{1 j} \operatorname{sign}^{a_{1 j}} e_{j}+k_{2 j} \operatorname{sign}^{a_{2 j}} \&, \quad j=\alpha, l
$$

where the parameters of the sliding mode surfaces satisfy the following relations: $k_{1 j}>0$, $k_{2 j}>0, \quad a_{2 j}<a_{1 j}$ and $1<a_{2 j}<2$. The symbol 'sign' represents a nonlinear function: $\operatorname{sign}^{p}(q)=\operatorname{sgn}(q)|q|^{p}$

To ensure the closed-loop stability of the formation system, the hybrid deployment controller is designed as follows:

$$
u_{j}=-f_{j}-\frac{1}{k_{2 j} a_{2 j}} \operatorname{sign}^{\left(2-a_{2 j}\right)} \&\left(1+k_{1 j} a_{1 j}\left|e_{j}\right|^{a_{1 j}-1}\right)-\hat{\delta}_{j} \operatorname{sgn}\left(s_{j}\right)-\kappa_{j} \operatorname{sgn}\left(s_{j}\right)-K_{j} s_{j}, j=\alpha, l
$$

where $K_{j}>0$ and $\kappa_{j}>0$, the term $-K_{j} s_{j}$ can improve the speed of approaching to the sliding surfaces, and $-\kappa_{j} \operatorname{sgn}\left(s_{j}\right)$ can enhance the anti-interference performance of the system. $\hat{\delta}_{j}$ are the estimated values of the upper bounds of perturbations, and are determined by the adaptive law proposed as follows:

$$
\delta_{j}^{\&}=\frac{1}{\varphi_{j}}\left|s_{j}\right| k_{2 j} a_{2 j}|\&|^{a_{2 j}-1}, j=\alpha, l
$$

where the parameter $\varphi_{j}>0$.

Theorem 2: For the tethered formation model shown in Eq. (10), if the deployment controller is proposed as Eq. (23), meanwhile the adaptive law for estimating the upper bounds of perturbations 
is designed as Eq. (23), then the HS-TSF can be deployed to the equilibrium state in finite time.

Proof: The proof will be conducted in two cases: $\&_{J} \neq 0$ and $\&=0$, respectively. For the first case $\& \neq 0$, the boundedness of the system could be illustrated by considering the following Lyapunov function:

$$
V_{j}=\frac{1}{2} s_{j}^{2}+\frac{1}{2} \varphi_{j}\left(\hat{\delta}_{j}-\delta_{j}\right)^{2}, \quad j=\alpha, l
$$

Differentiate the Lyapunov function with respect to the dimensionless time:

$$
\begin{aligned}
V_{j} & =s_{j} \&+\varphi_{j}\left(\hat{\delta}_{j}-\delta_{j}\right) \delta_{j}^{\&} \\
& =s_{j}\left(\underset{j}{\&}+k_{1 j} a_{1 j}\left|e_{j}\right|^{a_{1 j}-1} \underset{j}{\&}+k_{2 j} a_{2 j}|\&|^{a_{2 j}-1}\left(f_{j}+g_{j}+u_{j}\right)\right)+\varphi_{j}\left(\hat{\delta}_{j}-\delta_{j}\right) \delta_{j}^{\&}
\end{aligned}
$$

Then, substitute the proposed controller in Eq. (23) into Eq. (26):

$$
\begin{aligned}
V_{j} & =s_{j} k_{2 j} a_{2 j}|\&|^{a_{2 j}-1}\left(g_{j}-\hat{\delta}_{j} \operatorname{sgn}\left(s_{j}\right)-\kappa_{j} \operatorname{sgn}\left(s_{j}\right)-K_{j} s_{j}\right)+\varphi_{j}\left(\hat{\delta}_{j}-\delta_{j}\right) \delta_{j}^{\&} \\
& =k_{2 j} a_{2 j}|\&|^{a_{2 j}-1}\left(g_{j} s_{j}-\hat{\delta}_{j}\left|s_{j}\right|-\kappa_{j}\left|s_{j}\right|-K_{j} s_{j}^{2}\right)+\varphi_{j}\left(\hat{\delta}_{j}-\delta_{j}\right) \delta_{j}^{\&}
\end{aligned}
$$

Eq. (27) can be further simplified by substituting the proposed adaptive law in Eq. (24):

$$
\begin{aligned}
\downarrow_{j} & =k_{2 j} a_{2 j}|\&|^{a_{2 j}-1}\left(g_{j} s_{j}-\hat{\delta}_{j}\left|s_{j}\right|-\kappa_{j}\left|s_{j}\right|-K_{j} s_{j}^{2}+\left(\hat{\delta}_{j}-\delta_{j}\right)\left|s_{j}\right|\right) \\
& =k_{2 j} a_{2 j}|\&|^{a_{2 j}-1}\left(g_{j} s_{j}-\kappa_{j}\left|s_{j}\right|-K_{j} s_{j}^{2}-\delta_{j}\left|s_{j}\right|\right) \\
& \leq k_{2 j} a_{2 j}|\&|^{a_{2 j}-1}\left(-\kappa_{j}\left|s_{j}\right|-K_{j} s_{j}^{2}\right)
\end{aligned}
$$

According to the value ranges of the parameters in Eqs. (22) and (23), it can be found that the term $k_{2 j} a_{2 j}|\&|^{a_{2 j}-1}>0$. Thus, $V_{j}^{\&} \leq 0$. From this, it can be concluded that the close-loop system can satisfy the Lyapunov stability condition and the function $V_{j}$ is bounded. Therefore, the sliding mode surface $s_{j}$ and the adaptive deviation $\hat{\delta}_{j}-\delta_{j}$ are also bounded. Taking the boundness of $\delta_{j}$ into consideration, there will be an upper boundary for the adaptive estimation 
$\hat{\delta}_{j}$, namely, $\hat{\delta}_{j} \leq \hat{\delta}_{j}^{*}$. Furthermore, the finite time stability of the sliding mode surface could be illustrated by introducing another Lyapunov function in Appendix B.

For the second case $\underset{J}{\&}=0$, substituting the deployment controller in Eq. (23) to the formation model in Eq. (10) yields directly:

$$
\begin{aligned}
& =f_{j}+u_{j}+g_{j} \\
& =-\frac{1}{k_{2 j} a_{2 j}} \operatorname{sign}^{\left(2-a_{2 j}\right)} \&\left(1+k_{1 j} a_{1 j}\left|e_{j}\right|^{a_{1 j}-1}\right)-\hat{\delta}_{j} \operatorname{sgn}\left(s_{j}\right)-\kappa_{j} \operatorname{sgn}\left(s_{j}\right)-K_{j} s_{j}+g_{j} \\
& =g_{j}-\hat{\delta}_{j} \operatorname{sgn}\left(s_{j}\right)-\kappa_{j} \operatorname{sgn}\left(s_{j}\right)-K_{j} s_{j}, \quad(j=l, \alpha)
\end{aligned}
$$

The parameter $\kappa_{j}$ could be selected to be a value greater than the estimation error of the upper boundary of the perturbation terms, that is, set $\kappa_{j}>\delta_{j}-\hat{\delta}_{j}$. By doing this, the following discussions can be made: (i) If $s_{j}>0$, Eq. (29) turns into $g_{j}-\hat{\delta}_{j}-\kappa_{j}-K_{j}\left|s_{j}\right| \leq-K_{j}\left|s_{j}\right|$, thus \& will decrease; (ii) If $s_{j}<0$, Eq. (29) becomes $=g_{j}+\hat{\delta}_{j}+\kappa_{j}+K_{j}\left|s_{j}\right| \geq K_{j}\left|s_{j}\right|$, which means the value of $\&$ will increase. From these discussions, we may come to the conclusion that the system wouldn't always stay at the points $\left(e_{j} \neq 0\right.$ and $\left.\underset{J}{\&}=0\right)$, namely, $\underset{J}{\&}=0$ is not an attractor. Moreover, it can be found that there exists a small vicinity of $\&=0$, i.e. $|\&| \leq o_{j}$, where $o_{j}$ is a small positive constant, and within the vicinity satisfies: $\leq-K_{j}\left|s_{j}\right|$ for $s_{j}>0$, and $\geq K_{j}\left|s_{j}\right|$ for $s_{j}<0$. Thus, the crossing of trajectories between two boundaries of the small vicinity $\left(|\&| \leq o_{j}\right)$ are fulfilled in finite time [29]. For the region outside the vicinity, the sliding mode surface would converge to zero in finite time according to the conclusion drawn for the first case.

In summary, the sliding mode surface $s_{j}=0$ can be reached in finite time from anywhere of 
the phase plane under the proposed deployment controller with the adaptive law. After that, the finite-time convergence of the system states will be presented.

When the system reaches the sliding surface, one obtains:

$$
e_{j}+k_{1 j} \operatorname{sign}^{a_{1 j}} e_{j}+k_{2 j} \operatorname{sign}^{a_{2 j}} \underset{j}{\&}=0, \quad j=\alpha, l
$$

Considering the system states satisfy $\operatorname{sgn}\left(e_{j}\right)=-\operatorname{sgn}(\&)$ when $s_{j}=0$, Eq. (30) becomes:

$$
|\&|=\left(\frac{\left|e_{j}\right|+k_{1 j}\left|e_{j}\right|^{a_{1 j}}}{k_{2 j}}\right)^{\frac{1}{a_{2 j}}}
$$

Eq. (31) can be rewritten as:

$$
d|\tau|=\left(\frac{k_{2 j}}{\left|e_{j}\right|+k_{1 j}\left|e_{j}\right|^{a_{1 j}}}\right)^{\frac{1}{a_{2 j}}} d\left|e_{j}\right|
$$

The convergence time of the system states is obtained by integrating the two sides of Eq. (32) simultaneously:

$$
\begin{aligned}
t_{j} & =\int_{0}^{\left|e_{j}(0)\right|}\left(\frac{k_{2 j}}{e_{j}+k_{1 j} e_{j}^{a_{1 j}}}\right)^{\frac{1}{a_{2 j}}} d e_{j} \\
& =\frac{a_{2 j}\left|e_{j}(0)\right|^{1-\frac{1}{a_{2 j}}}}{k_{1 j}\left(a_{2 j}-1\right)} \cdot F\left(\frac{1}{a_{2 j}}, \frac{a_{2 j}-1}{a_{2 j}\left(a_{2 j}-1\right)}, 1+\frac{a_{2 j}-1}{a_{2 j}\left(a_{2 j}-1\right)},-k_{1 j}\left|e_{j}(0)\right|^{a_{1 j}-1}\right)
\end{aligned}
$$

where $F(\bullet)$ represents the Gauss's Hypergeometric function [31].

Hence, Theorem 2 is proven completely, which means that the proposed hybrid deployment controller shown in Eq. (23) and the adaptive law in Eq. (24) can ensure that the hub-spoke formation system can be deployed to the desired equilibrium state in finite time. 
Remark 3: Taking thrust as one of the control forces could improve control accuracy for the libration motion of tethers, thereby effectively reducing the risk of tether entanglement. The hybrid tension-thrust controller proposed based on the nonsingular TSMC method is able to deploy tethers fully in finite time without singularity of the control input. Besides, in this section an adaptive law is also developed to enhance the tolerance of the control system to the inherent perturbations.

\section{Numerical Simulations and Discussions}

In this section the effectiveness of the proposed two deployment controllers is validated by numerical simulations. All simulations were performed in MATLAB/SIMULINK environment.

\subsection{Numerical Simulation Settings}

The orbit altitude of the HS-TSF in simulation studies is set as $h=1000 \mathrm{~km}$. The central satellite is assumed as a uniform sphere with the radius $r=1 \mathrm{~m}$ and mass $m_{c}=300 \mathrm{~kg}$. Thus, the inertial moment of the central body is $J=0.4 m_{c} r^{2}$. The desired stable spinning velocity of the central body is set as one hundred times faster than the orbital angular velocity, that is $N=100$. The mass of each sub-satellite is $m_{i}=10 \mathrm{~kg}$. The expected tether length is set as $l_{\text {eq }}=100 \mathrm{~m}$. Thus,

the system states at the final time of deployment are $\boldsymbol{x}_{\mathrm{eq}}=[0,0,100,0]^{\mathrm{T}}$. Given three sets of initial states $\quad \boldsymbol{x}_{0}=[-0.1,0,1,0]^{\mathrm{T}},[-0.15,0,5,0]^{\mathrm{T}},[-0.2,0,10,0]^{\mathrm{T}}$, simulations are carried out to demonstrate the deployment of the formation system with different initial tether lengths and libration angles.

The parameter of the term introduced in the dynamic model Eq. (11) is $\varepsilon=5$. The parameters of the sliding mode surfaces for the tension deployment controller are selected as $c_{1}=10000$ and $c_{2}=200$, which could be obtained by assigning the poles of the matrix $\boldsymbol{A}_{e}$ to the point 
$(-100,0)$. The selection of other parameters of the controller follows the relationships declared in Section 3. The parameter in controller Eq. (14) is chosen as $\eta=5$. The parameter in Eq. (15) is $\gamma=2000$, and the initial integral value for the adaptive law is $\hat{\delta}_{D}(0)=8$.

For the tension-thrust deployment controller, the parameters of the sliding surfaces are $k_{1 \alpha}=45, k_{1 l}=2, k_{2 \alpha}=15, k_{2 l}=4$. Since the magnitude of tether length is much larger than that of libration angle, the parameters $k_{1 \alpha}, k_{2 \alpha}$ should be larger than $k_{1 l}, k_{2 l}$. From the form of the controller shown in Eq. (23), the relationships $a_{1 j}>a_{2 j}$ and $1<a_{2 j}<2$ could ensure the non-singularity of the controller, so these parameters are chosen as: $a_{1 \alpha}=a_{1 l}=3$ and $a_{2 \alpha}=a_{2 l}=1.5$. The other involved parameters in Eq. (23) are selected as: $K_{\alpha}=K_{l}=0.1, \kappa_{\alpha}=1$ and $\kappa_{l}=3$. When selecting the parameter $K_{j}$, it needs to be noted that although a larger value can increase the approaching velocity to the sliding surface, the chattering effect on the control system might be stronger. The adaptive parameters in Eq. (24) are $\varphi_{\alpha}=0.01$ and $\varphi_{l}=400$, and the initial integral values for the adaptive law are $\delta_{\alpha}(0)=5$ and $\delta_{l}(0)=150$.

In addition, for the purpose of reducing the chattering on the control systems, the switching function $\operatorname{sgn}(s)$ in the proposed two controllers is replaced by a hyperbolic tangent function $\tanh (s / \xi)$, where the parameter is selected as $\xi=0.01$. Besides, $u_{l}$ and $u_{\alpha}$ are transformed into tether tension $T$ and thrust force $F_{t}$ respectively in order to make the control inputs more intuitive.

\subsection{Simulation Results}

(I) Simulations with different initial system states 
First of all, Figs. 4-7 depict the system states during the deployment under the tension and tension-thrust control strategies, respectively. The selected three sets of initial states are denoted as $x(1), x(2), x(3)$ in the figures. Overall, it can be seen that the proposed two controllers can deploy the sub-satellites to the equilibrium state successfully, although the initial tether length and libration angle differs. As shown in Figs. 4 and 5, the tension-thrust controller provides a faster deployment process than the tension controller: the time required under the former controller is about 0.5 orbits, while under the other is about 0.7 orbits. The maximum deployment velocity during the process is about $0.2 \mathrm{~m} / \mathrm{s}$ and $0.05 \mathrm{~m} / \mathrm{s}$, respectively. Figures. 6 and 7 show that the libration angle under the tension-thrust controller converges directly from the given initial values to zero, while under the tension controller the libration angle firstly increases till the maximum, which is nearly $1.5 \mathrm{rad}$, and then swings back toward the equilibrium state. Although there wouldn't entanglement between tethers and the central body under the two controllers, the risk of tether entanglement is obviously larger without thrust. Besides, Fig. 5 and Fig. 7 show that the curves of tether length rate and libration angle rate fluctuate slightly around zero after the tethers are deployed completely, which is partly caused by the persistent periodic perturbations existed in the system. And it is worth noting that the fluctuation of the libration angle rate is smaller under the hybrid tension-thrust controller. 


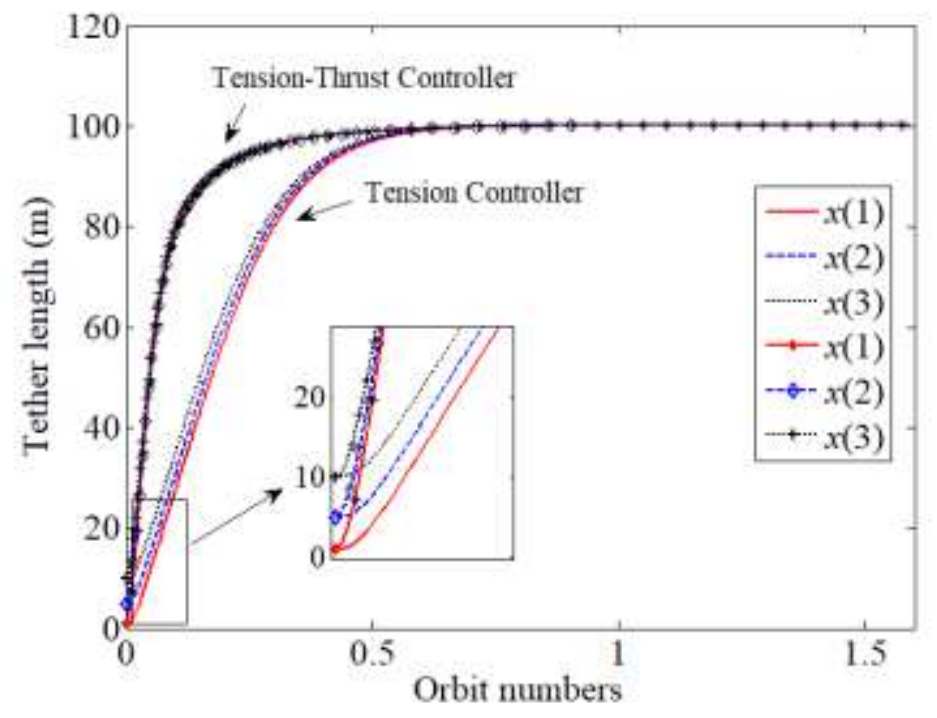

Fig. 4. The time histories of the tether length.

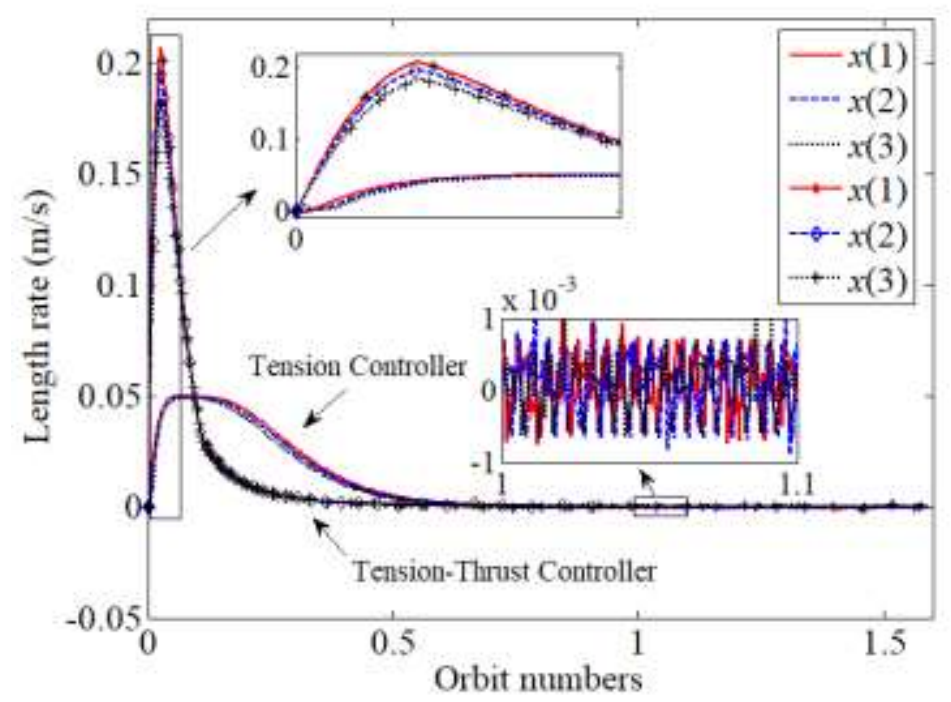

Fig. 5. The time histories of the tether length rate. 


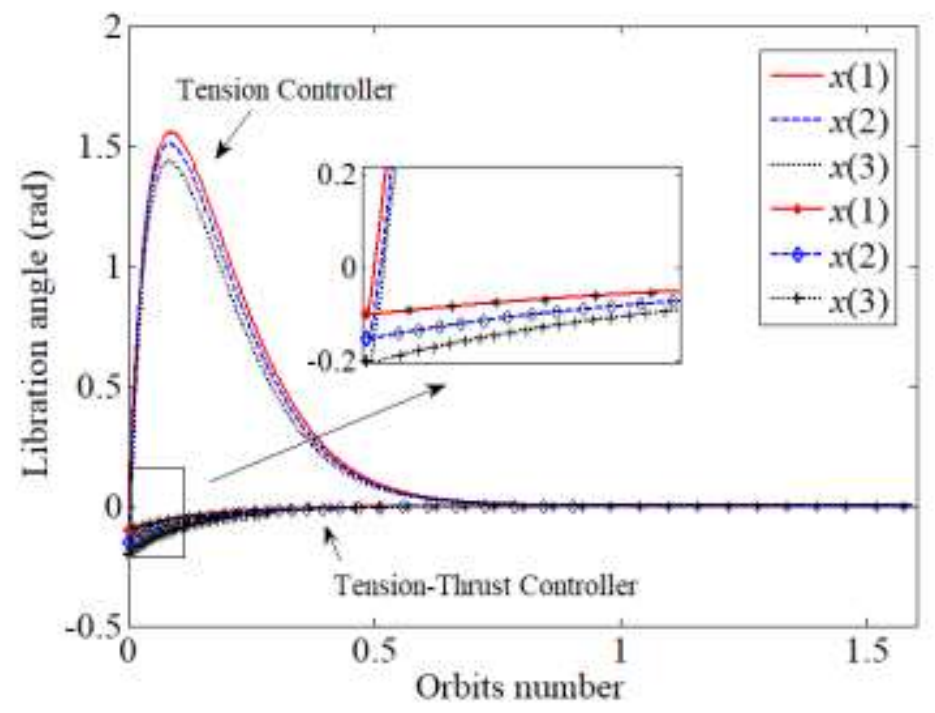

Fig. 6. The time histories of the libration angle.

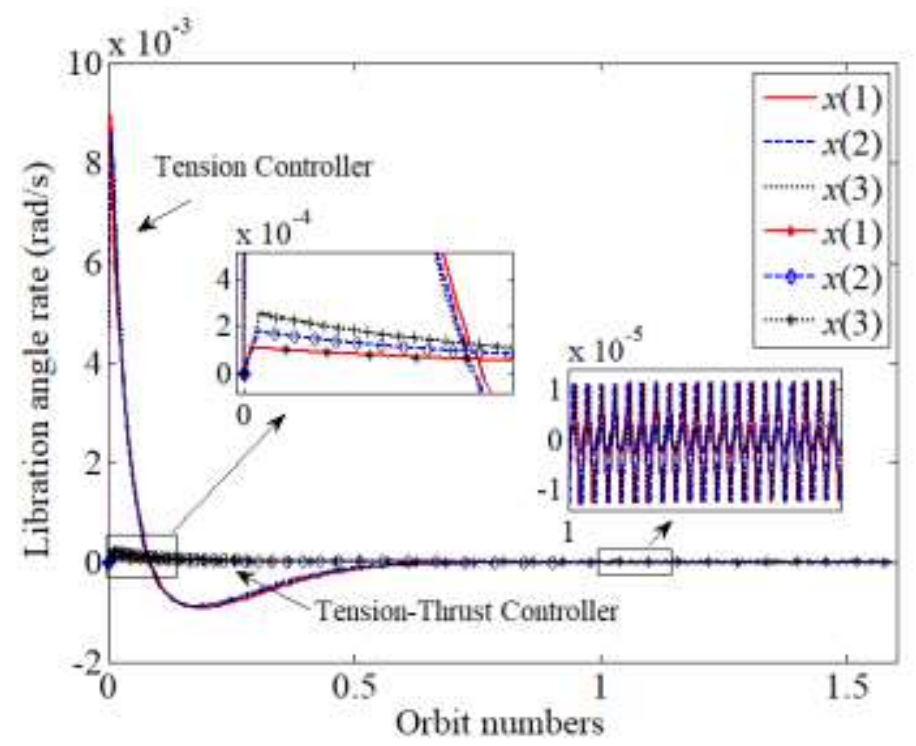

Fig. 7. The time histories of the libration angle rate.

The control inputs are shown in Figs. 8 and 9. The tether tension in both controllers is always larger than zero and eventually stabilize at about $10.3 \mathrm{~N}$, which means that there is no slack in the tethers during deployment process. The thrust force increases rapidly to about $0.4 \mathrm{~N}$ at the initial moment of deployment to stabilize the libration angle, and gradually converges to zero as the tethers are fully deployed. 


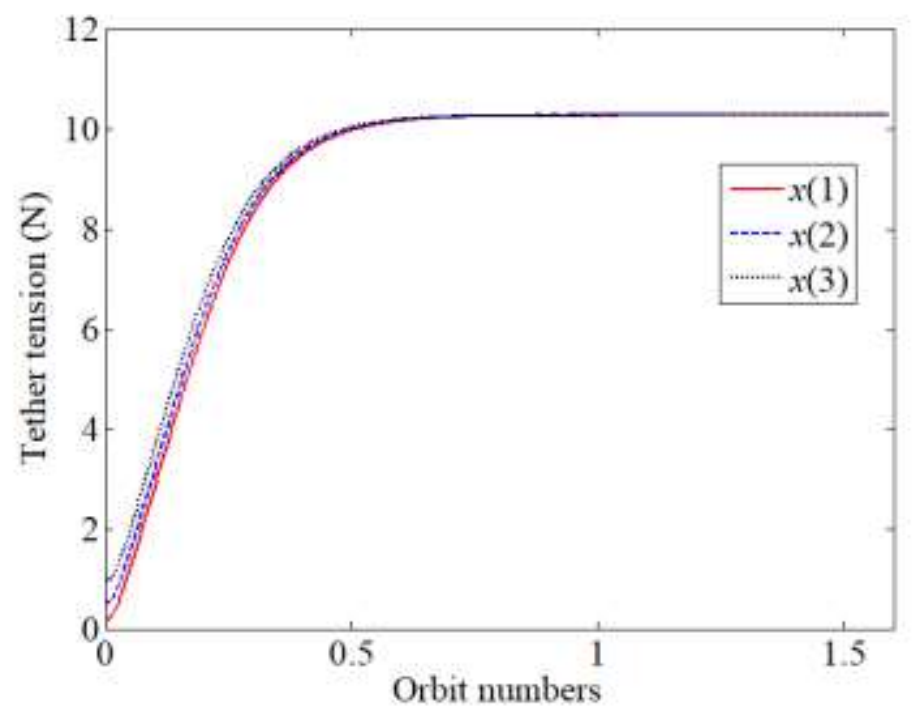

Fig. 8. The time histories of the control input for the tension controller.

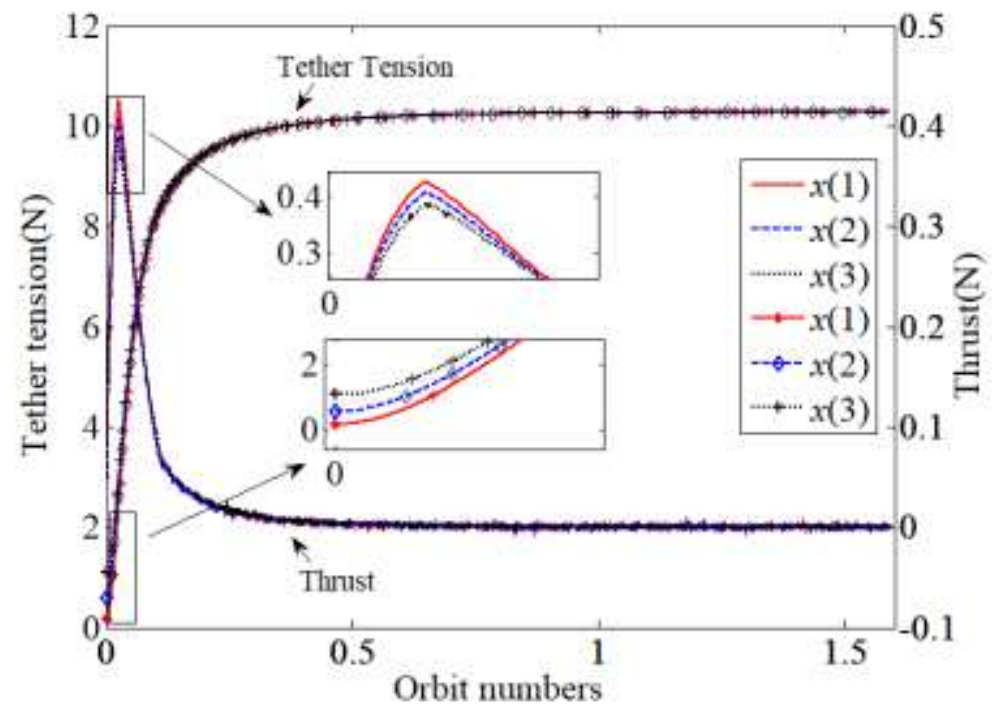

Fig. 9. The time histories of the control inputs for the tension-thrust controller.

Next, it is interesting to see the system energy variation during the deployment process under the proposed two control strategies. Subtracting the orbital energy from the total energy shown in Eqs. (3) and (4) yields the relative kinetic and potential energy of the HS-TFS:

$$
\bar{T}=\frac{1}{2} \sum_{i=1}^{n} m_{i} v_{i}^{2}+\frac{1}{2} J(\omega+\Omega)^{2}
$$




$$
\bar{U}=\mu_{e} \sum_{i=1}^{n} \frac{m_{i}}{2 R^{3}}\left(\boldsymbol{p}_{i} \cdot \boldsymbol{p}_{i}-3\left(\boldsymbol{i} \cdot \boldsymbol{p}_{i}\right)^{2}\right)
$$

Thus, the total relative energy of the HS-TSF is $E_{r}=\bar{T}+\bar{U}$. The curves of the relative kinetic energy, potential energy and the total relative energy are shown in Figs. 10-12. The values of $\bar{T}$ gradually increases to about 2080 under the proposed two controllers, while $\bar{U}$ decreases to -0.11 . After the tethers are fully deployed, the values of $\bar{T}$ and $\bar{U}$ remain nearly constants. Besides, since the magnitude of the relative potential energy is much smaller than that of the relative kinetic energy, the curves of $\bar{T}$ and $E_{r}$ are almost the same.

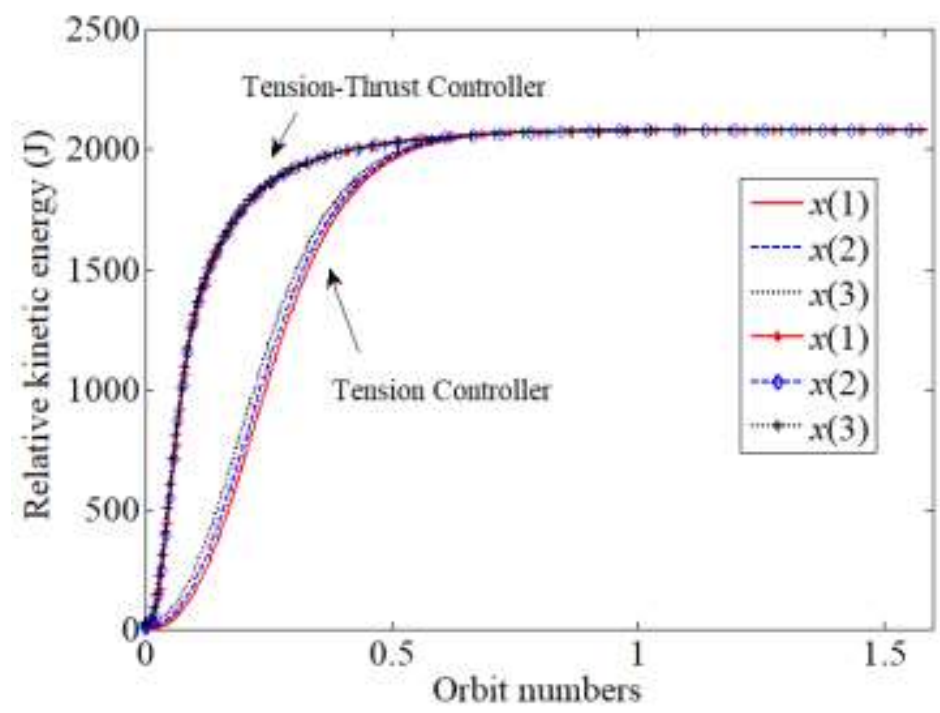

Fig. 10. The time histories of the relative kinetic energy. 


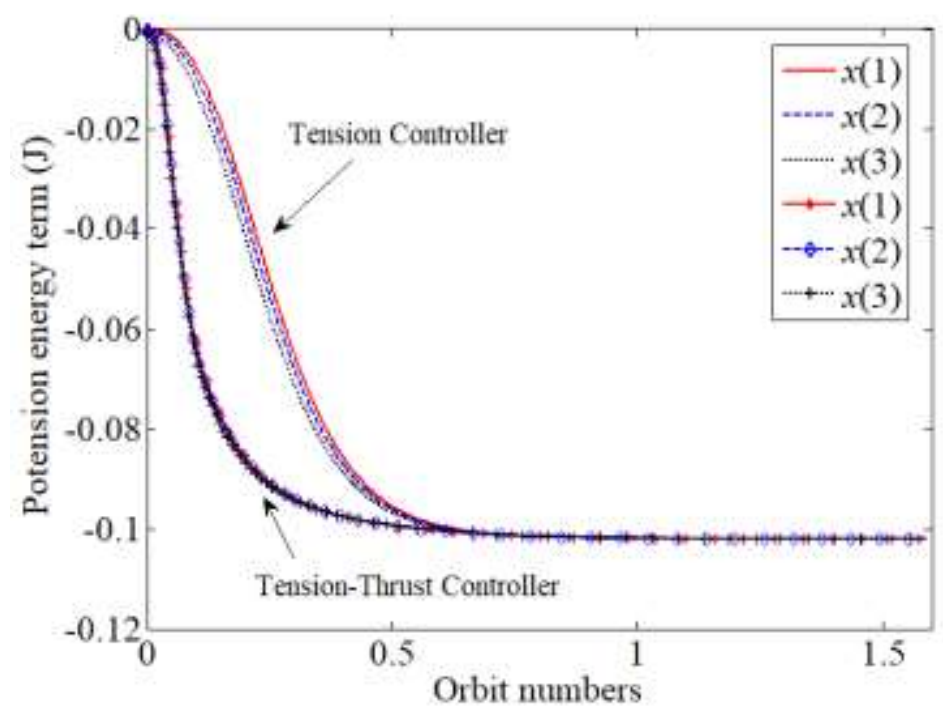

Fig. 11. The time histories of the potential energy term.

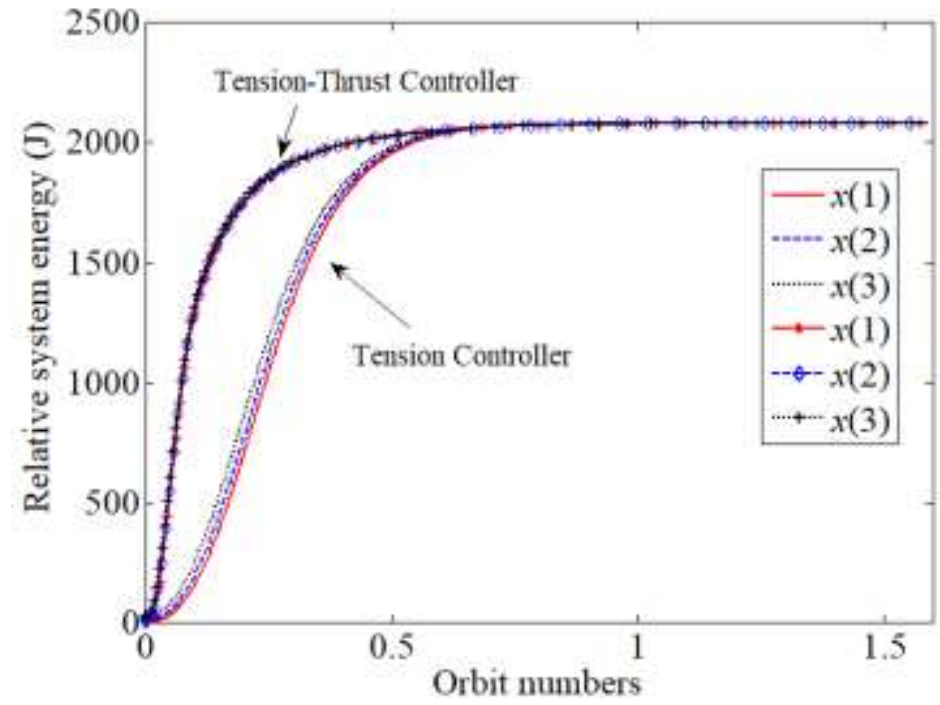

Fig. 12. The time histories of the relative system energy.

The above results demonstrate that both the proposed controllers are capable of deploying the formation system in different initial states. Next, take the first set of the initial states $x(1)$ as an example to reveal the configuration changes of the formation system during deployment, as shown in Figs. 13-16. The units of the axes in the subgraphs of these figures are meter $(\mathrm{m})$. The central satellite is drawn in black, and the four lines with different colors represent tethers, denoted by 1 , 
2, 3 and 4, respectively. The configuration changes are recorded and presented per 0.1 orbits number. Figures 13 and 15 show the positional relationship between tethers and the central body during deployment. It can be seen there is no tether entanglement under the two proposed controllers. Figures 14 and 16 show that the tethers are gradually deployed until the expected final length $(100 \mathrm{~m})$ is reached.
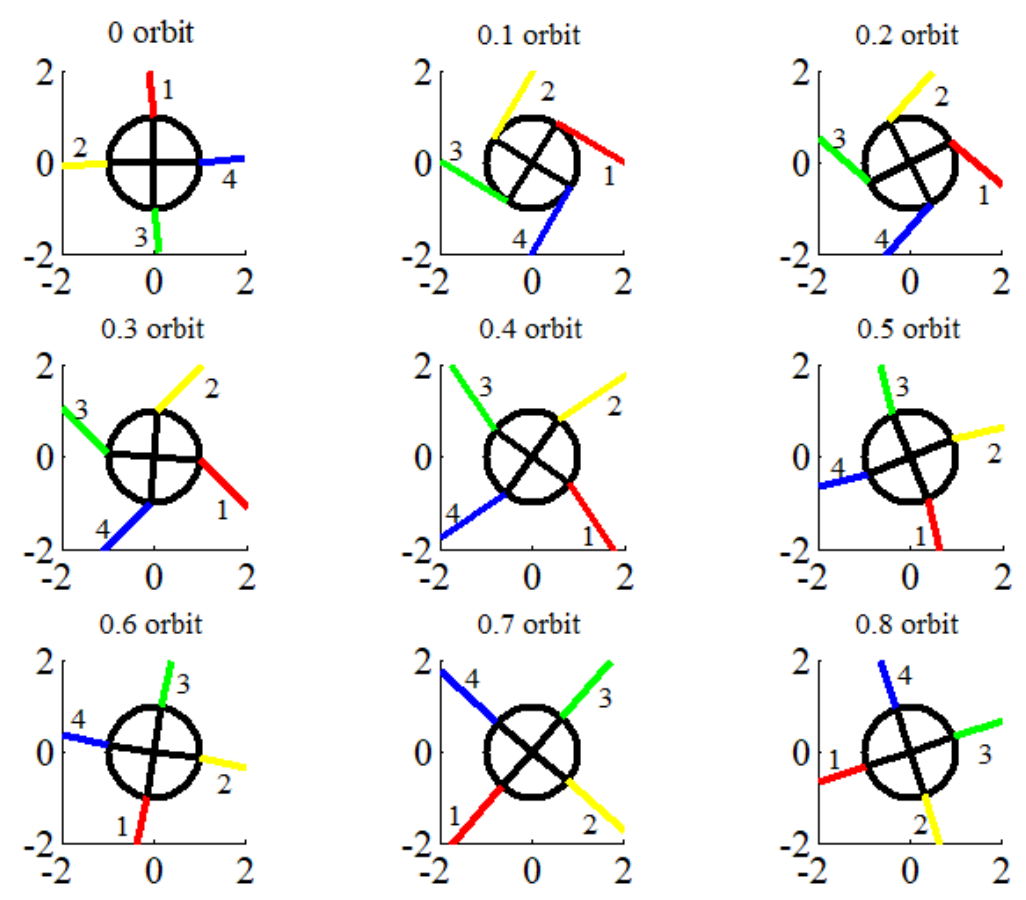

Fig. 13. Positional relations between tethers and the central body under tension controller. 

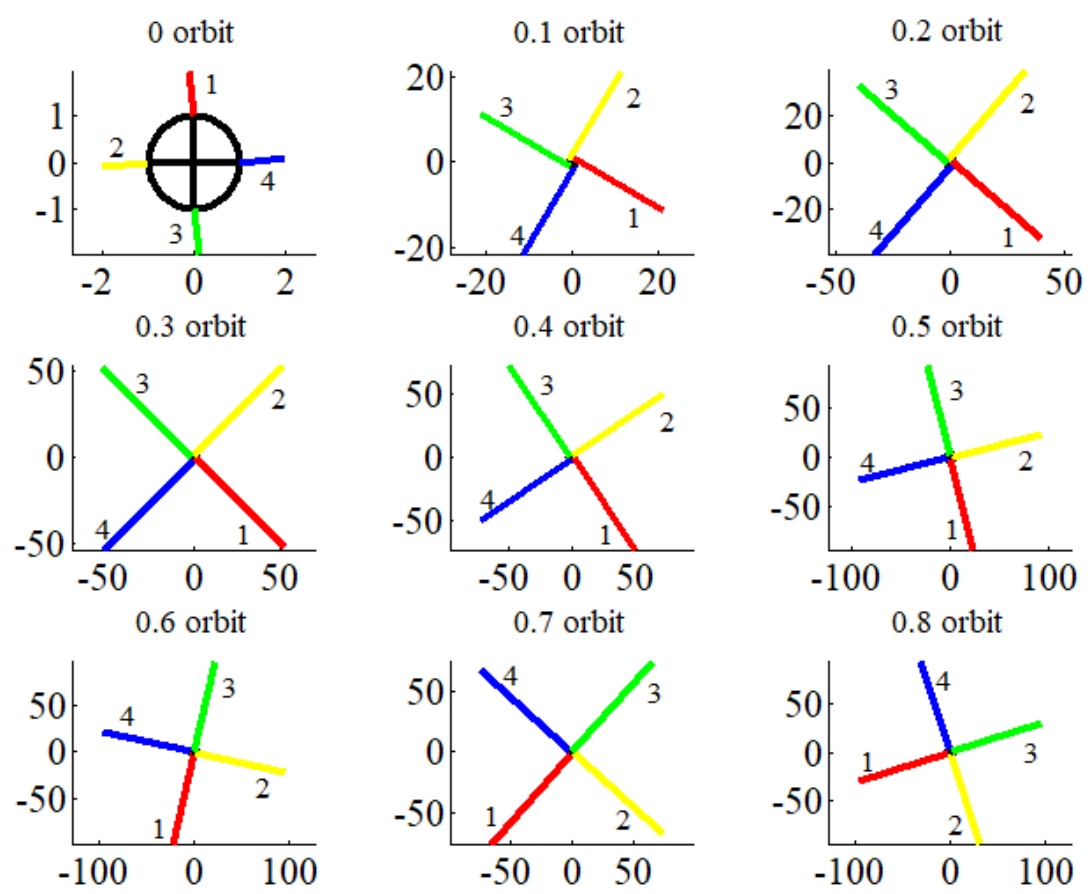

Fig. 14. Configuration changes under tension controller.
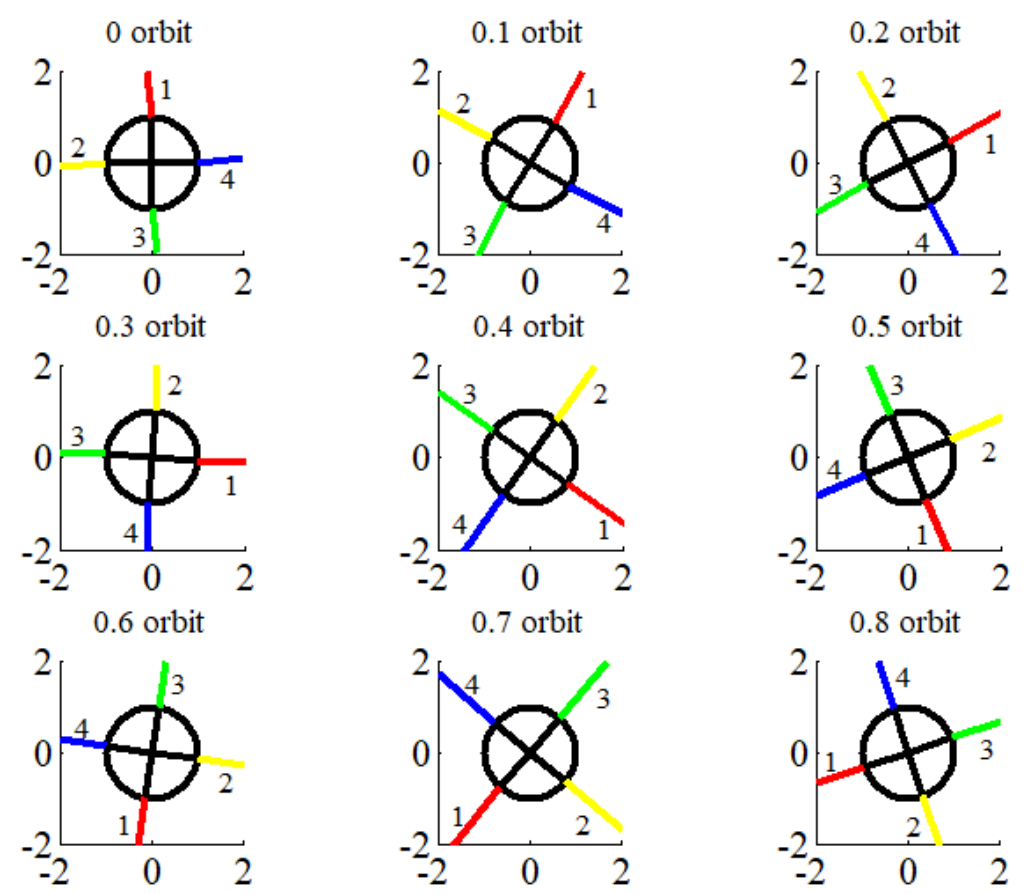

Fig. 15. Positional relations between tethers and the central body under tension-thrust controller. 

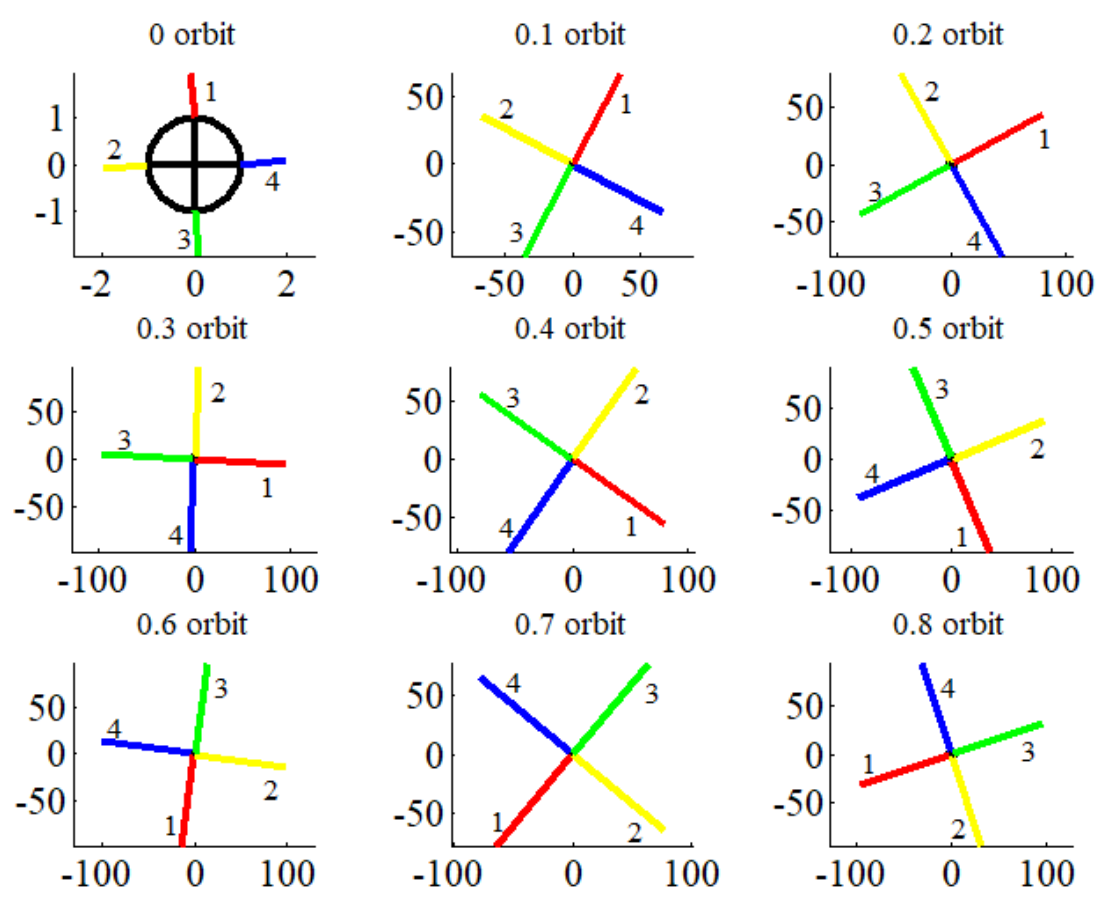

Fig. 16. Configuration changes under tension-thrust controller.

Figure 17 shows the deployment trajectories of the sub-satellites in orbital coordinate system under two control strategies. Intuitively, the inner circles of the trajectories are sparser than the outer circles, which reflects that the deployment velocities are faster in the initial stage under the controllers, as shown in Fig. 5. 


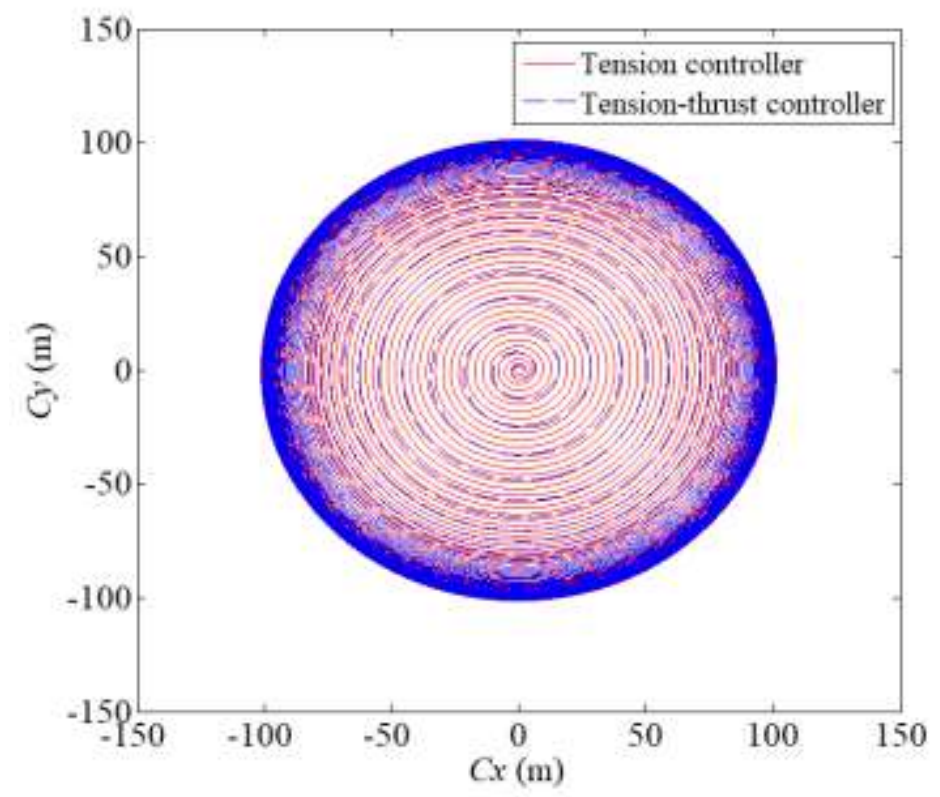

Fig. 17. Deployment trajectories in orbital coordinate system.

(II) Simulations with different spin rates of the central satellite

As one might expect, the spin rate of the central body has an impact on the deployment of the formation system. To reveal this impact, as well for the purpose of showing the validity of the proposed controllers, it is necessary to illustrate the deployment states with different spin rates of the central body. The spin rates of the central body are chosen as: $N=100,110$ and 120, respectively. Without loss of generality, the initial system state here is set as $x(1)$.

It can be seen from the dynamic model of the formation system, shown in Eq. (10), that a higher spin rate of the central body will result in a higher frequency of the inherent periodic perturbations. It is expected that the proposed controllers combined with adaptive laws could deal with the influence of the perturbations and thus improve the anti-disturbance ability of the system. The simulations results, shown in Figs. 18 and 19, indicate that the formation system is successfully deployed under the presented controllers despite the spin rate varies. When using the tension controller, if the spin rate of the central satellite increases, the time required for the 
deployment process decreases. And the maximum libration angle also slightly decreases as tethers are deployed from the central body spinning at a higher speed. While the deployment process is almost unchanged with the different spin rate when the tension-thrust controller is employed in system. This indicates that the deployment under the hybrid controller is less sensitive to changes of the spin rate.

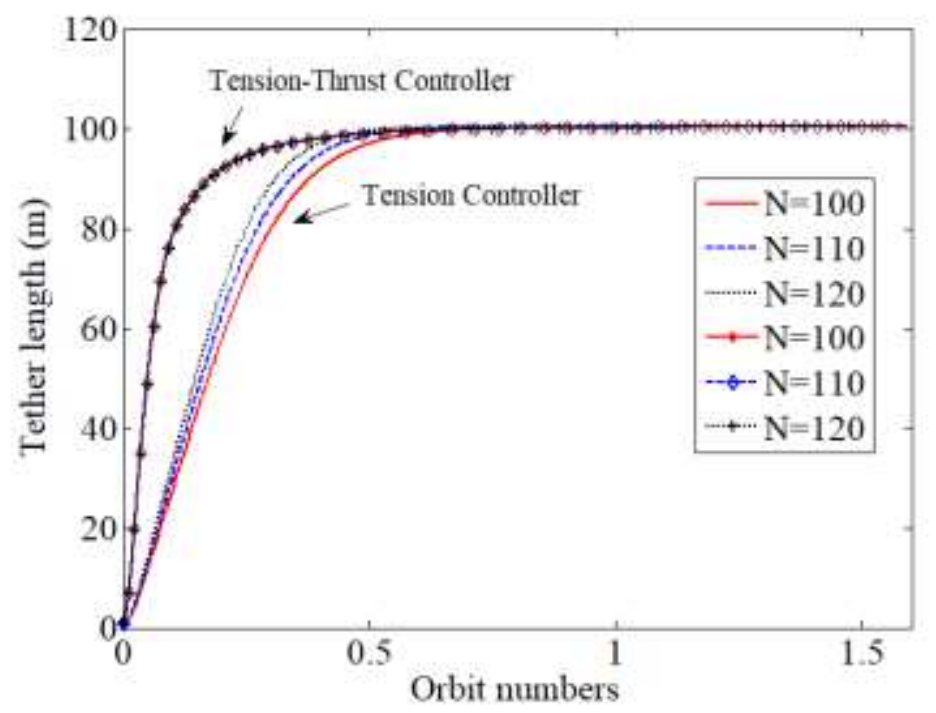

Fig.18. The time histories of the tether length.

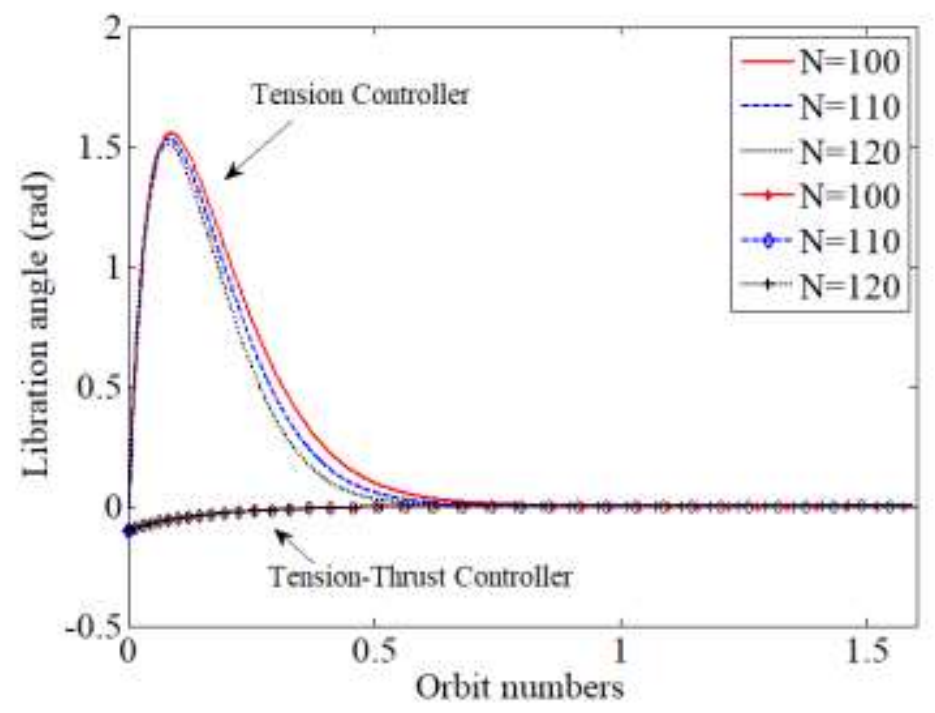

Fig. 19. The time histories of the libration angle. 
The control inputs of the proposed controllers are shown in Figs. 20 and 21. It is clear from the figures that a larger control tension is required to deploy the formation system into a configuration with a relatively high rotation rate. This may be explained from the aspect of the centrifugal forces generated by the rotation of the system. In addition, when it comes to the hybrid controller, if the central body spins at a higher rate, the maximum thrust during the deployment process will becomes larger, as shown in Fig. 21, for the reason that a greater thrust force is needed to provide rotational tangential acceleration.

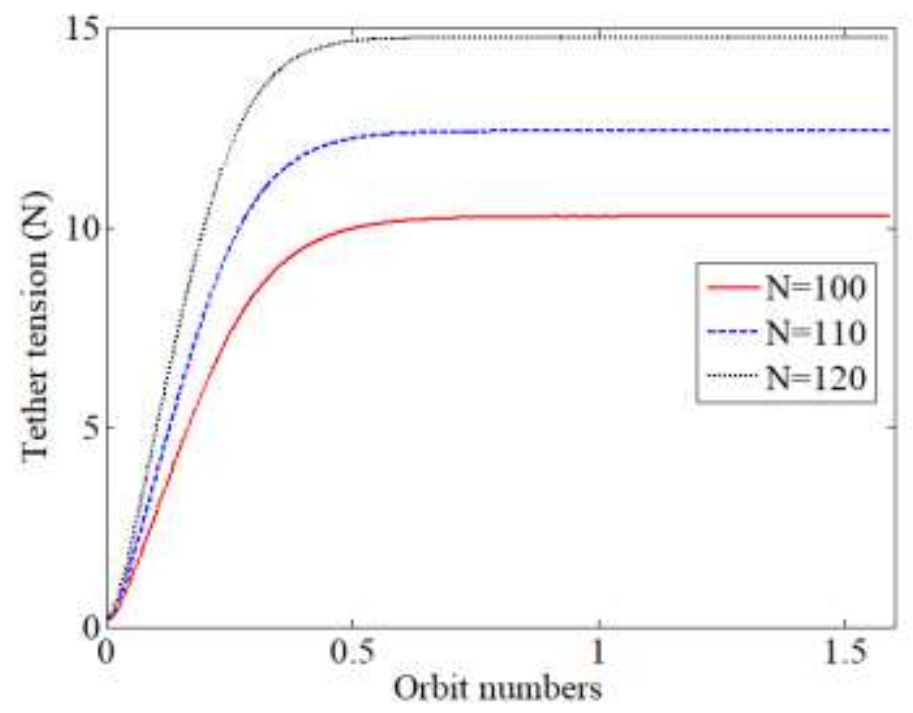

Fig. 20. The time histories of the control input for tension controller. 


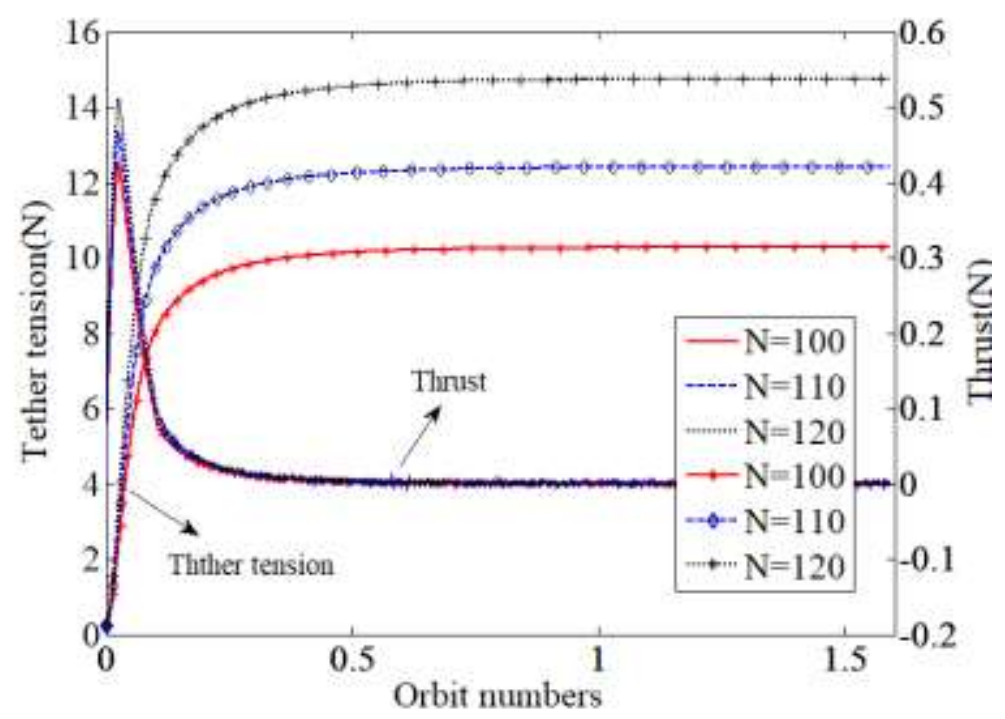

Fig. 21. The time histories of the control inputs for tension-thrust controller.

\section{Conclusions}

Deployment control of a spinning HS-TSF system has been studied. The dynamic model, treating the central satellite as a rigid body and the sub-satellites as point masses, was used for controller design. First, a tension controller based on the nonlinear underactuated SMC method was derived, enabling to deploy the HS-TSF system to the desired configuration, which shows the possibility of controlling the deployment of a spinning HS-TSF without consuming fuel of the subsatellites. Then, a hybrid controller that takes tether tensions and thrusts as control inputs was obtained using the nonsingular TSMC method. In addition, adaptive laws were introduced in the controller design to estimate the unknown upper bounds of perturbations. Using the Lyapunov stability analysis, the closed-loop system of the HS-TSF is stable in a neighborhood of the equilibrium state under the tension controller, while the tension-thrust controller could guarantee the finite-time stability of the system. The simulation case studies show the validity of the proposed controllers in different initial system states and different spin rates of the central satellite. The results reveal that successful deployment of the HS-TSF can be obtained by either only tension or 
hybrid controller. In particular, the hybrid tension-thrust controller shows advantages when the demands for safety and rapidity are more concerned, because it demonstrates better performance in terms of deployment velocity and reducing the risk of tether entanglement.

\section{Declarations}

\subsection{Funding}

The authors gratefully acknowledge the support of the Fundamental Research Funds from the

National Natural Science Foundation of China (No. 62111530051), China Scholarship Council Scholarship (No. 201906290212), and the Discovery Grant (RGPIN-2018-05991) of the Natural Sciences and Engineering Research Council of Canada.

\subsection{Conflicts of interest/Competing interests}

The authors declare no conflict of interest in this article.

\subsection{Availability of data and material}

Data sharing not applicable to this article as no datasets were generated or analyzed during the current study.

\subsection{Code availability (software application or custom code)}

The custom codes in the current study are available from the corresponding author on reasonable request.

\subsection{Authors' contributions}

Mr. Chenguang Liu 
Conceptualization: Lead; Formal analysis: Equal; Funding acquisition: Supporting; Investigation: Equal; Methodology: Lead; Software: Lead; Validation: Equal; Writing - original draft: Lead; Writing - review \& editing: Supporting.

Dr. Wei Wang

Conceptualization: Equal; Funding acquisition: Lead; Investigation: Supporting; Project administration: Lead; Resources: Lead; Supervision: Lead; Writing - review \& editing: Supporting.

Dr. Junjie Kang

Methodology: Supporting; Software: Supporting; Writing - original draft: Supporting.

Dr. Zheng H. Zhu

Conceptualization: Equal; Formal analysis: Equal; Funding acquisition: Lead; Investigation: Lead;

Methodology: Equal; Project administration: Lead; Resources: Lead; Software: Supporting; Supervision: Lead; Validation: Equal; Writing - original draft: Equal; Writing - review \& editing: Lead.

\subsection{Ethics approval}

Not applicable.

\subsection{Consent to participate}

Not applicable. 


\subsection{Consent for publication}

Not applicable.

\section{Appendix A}

The Lyapunov function for the formation system Eq. (11) is chosen as:

$$
V=\frac{1}{2} s^{2}+\frac{1}{2} \gamma \delta_{D}^{8 / 2}
$$

Perform the differentiation of the Lyapunov function with respect to the dimensionless time:

$$
\begin{aligned}
I^{\&} & =s \&+\gamma \delta_{D}^{\&} \delta_{D}^{\&} \\
& =s\left(c_{1} x_{2}+c_{2} \bar{f}_{\alpha}+c_{2} \bar{g}_{\alpha}+\frac{\partial \bar{f}_{\alpha}}{\partial x_{1}} x_{2}+\frac{\partial \bar{f}_{\alpha}}{\partial x_{2}}\left(\bar{f}_{\alpha}+\bar{g}_{\alpha}\right)+\frac{\partial \bar{f}_{\alpha}}{\partial x_{3}} x_{4}+\frac{\partial \bar{f}_{\alpha}}{\partial x_{4}}\left(f_{l}+g_{l}+u_{l}\right)\right)+\gamma \delta_{D} \delta_{D}^{\&}
\end{aligned}
$$

Substituting the deployment controller Eq. (14) into Eq. (A2) yields:

$$
\begin{aligned}
I^{\&} & =s\left(c_{2} \bar{g}_{\alpha}+\frac{\partial \bar{f}_{\alpha}}{\partial x_{2}} \bar{g}_{\alpha}+\frac{\partial \bar{f}_{\alpha}}{\partial x_{4}} g_{l}-\hat{\delta}_{D} \operatorname{sgn}(s)-\eta s\right)+\gamma \delta_{D}^{\delta} \delta_{D}^{\&} \\
& =-\eta s^{2}-\hat{\delta}_{D}|s|+s\left(c_{2} \bar{g}_{\alpha}+\frac{\partial \bar{f}_{\alpha}}{\partial x_{2}} \bar{g}_{\alpha}+\frac{\partial \bar{f}_{\alpha}}{\partial x_{4}} g_{l}\right)+\gamma \delta_{D}^{\delta} \delta_{D}^{\&} \\
& \leq-\eta s^{2}-\hat{\delta}_{D}|s|+|s| \delta_{D}+\gamma \delta_{D}^{\&} \delta_{D}^{\&} \\
& =-\eta s^{2}-\delta_{D}^{\delta}|s|+\gamma \delta_{D}^{\delta} \delta_{D}^{\&}
\end{aligned}
$$

Then, substituting the adaptive law Eq. (15) into Eq. (A3) leads to:

$$
I^{\&} \leq-\eta s^{2}-\delta_{D}^{\&}|s|+\gamma \delta_{D}^{\&} \frac{1}{\gamma}|s| \leq-\eta s^{2}
$$

Thus, the control system asymptotically converges to the sliding mode surface $s=0$.

\section{Appendix B}

In order to demonstrate the finite time stability of the sliding mode surface, consider the 
following Lyapunov function:

$$
V_{j}^{*}=\frac{1}{2} s_{j}^{2}+\frac{1}{2} \psi_{j}\left(\hat{\delta}_{j}-\hat{\delta}_{j}^{*}\right)^{2}, \quad j=\alpha, l
$$

where the parameter $\psi_{j}$ satisfies $\psi_{j}>\varphi_{j}$.

Differentiating the Lyapunov function and substituting the proposed controller shown in Eq.

(23) leads to:

$$
\begin{aligned}
& V_{j}^{\&}=k_{2 j} a_{2 j}|\&|^{a_{2 j}-1}\left(g_{j} s_{j}-\hat{\delta}_{j}\left|s_{j}\right|-\kappa_{j}\left|s_{j}\right|-K_{j} s_{j}^{2}\right)+\psi_{j}\left(\hat{\delta}_{j}-\hat{\delta}_{j}^{*}\right) \delta_{j}^{\&} \\
& \quad \leq k_{2 j} a_{2 j}|\&|^{a_{j j}-1}\left(\delta_{j}\left|s_{j}\right|-\hat{\delta}_{j}\left|s_{j}\right|-\kappa_{j}\left|s_{j}\right|-K_{j} s_{j}^{2}\right)+\psi_{j}\left(\hat{\delta}_{j}-\hat{\delta}_{j}^{*}\right) \delta_{j}^{\&}
\end{aligned}
$$

Then, substituting the adaptive law in Eq. (24) into Eq. (B2) yields:

$$
\begin{aligned}
V_{j}^{\&} & \leq k_{2 j} a_{2 j}|\&|^{a_{2 j}-1}\left(\delta_{j}\left|s_{j}\right|-\hat{\delta}_{j}\left|s_{j}\right|-\kappa_{j}\left|s_{j}\right|-K_{j} s_{j}^{2}\right)+\frac{\psi_{j}}{\varphi_{j}}\left(\hat{\delta}_{j}-\hat{\delta}_{j}^{*}\right)\left|s_{j}\right| k_{2 j} a_{2 j}|\&|^{a_{2 j}-1} \\
& \leq k_{2 j} a_{2 j}|\&|^{a_{2 j}-1}\left(\delta_{j}\left|s_{j}\right|-\hat{\delta}_{j}\left|s_{j}\right|-\kappa_{j}\left|s_{j}\right|+\frac{\psi_{j}}{\varphi_{j}}\left(\hat{\delta}_{j}-\hat{\delta}_{j}^{*}\right)\left|s_{j}\right|\right) \\
& \leq k_{2 j} a_{2 j}|\&|^{a_{2 j}-1}\left(\delta_{j}\left|s_{j}\right|-\hat{\delta}_{j}\left|s_{j}\right|+\hat{\delta}_{j}^{*}\left|s_{j}\right|-\hat{\delta}_{j}^{*}\left|s_{j}\right|-\kappa_{j}\left|s_{j}\right|+\frac{\psi_{j}}{\varphi_{j}}\left(\hat{\delta}_{j}-\hat{\delta}_{j}^{*}\right)\left|s_{j}\right|\right) \\
& \leq k_{2 j} a_{2 j}|\&|^{a_{2 j}-1}\left(-\left(\hat{\delta}_{j}^{*}-\delta_{j}+\kappa_{j}\right)\left|s_{j}\right|+\left(\frac{\psi_{j}}{\varphi_{j}}-1\right)\left(\hat{\delta}_{j}-\hat{\delta}_{j}^{*}\right)\left|s_{j}\right|\right)
\end{aligned}
$$

Considering that $\hat{\delta}_{j}<\hat{\delta}_{j}^{*}$, Eq. (B3) can be written as:

$$
V_{j}^{\&} \leq k_{2 j} a_{2 j}|\&|^{a_{j}-1}\left(-\left(\hat{\delta}_{j}^{*}-\delta_{j}+\kappa_{j}\right)\left|s_{j}\right|-\left(\frac{\psi_{j}}{\varphi_{j}}-1\right)\left|\hat{\delta}_{j}-\hat{\delta}_{j}^{*}\right|\left|s_{j}\right|\right)
$$

Further rewrite Eq. (B4) as follows:

$$
v_{j}^{\&} \leq-\Delta_{1 j}\left|s_{j}\right|-\Delta_{2 j}\left|\hat{\delta}_{j}-\hat{\delta}_{j}^{*}\right|
$$

with 


$$
\begin{aligned}
& \Delta_{1 j}=\left.k_{2 j} a_{2 j}|\&|\right|^{a_{2 j}-1}\left(\hat{\delta}_{j}^{*}-\delta_{j}+\kappa_{j}\right) \\
& \Delta_{2 j}=k_{2 j} a_{2 j}|\&|^{a_{j j}-1}\left(\frac{\psi_{j}}{\varphi_{j}}-1\right)\left|s_{j}\right|
\end{aligned}
$$

It is easy to find that the terms $\Delta_{1 j}, \Delta_{2 j}$ satisfy $\Delta_{1 j} \geq 0, \Delta_{2 j} \geq 0$.

Hence,

$$
\begin{aligned}
V_{j}^{\&} & \leq-\sqrt{2} \Delta_{1 j} \frac{\left|s_{j}\right|}{\sqrt{2}}-\frac{\sqrt{2} \Delta_{2 j}}{\sqrt{\psi_{j}}} \frac{\left|\hat{\delta}_{j}-\hat{\delta}_{j}^{*}\right| \sqrt{\psi_{j}}}{\sqrt{2}} \\
& \leq-\min \left(\sqrt{2} \Delta_{1 j}, \frac{\sqrt{2} \Delta_{2 j}}{\sqrt{\psi_{j}}}\right)\left(\frac{\left|s_{j}\right|}{\sqrt{2}}+\frac{\left|\hat{\delta}_{j}-\hat{\delta}_{j}^{*}\right| \sqrt{\psi_{j}}}{\sqrt{2}}\right) \\
& \leq-\min \left(\sqrt{2} \Delta_{1 j}, \frac{\sqrt{2} \Delta_{2 j}}{\sqrt{\psi_{j}}}\right)\left(\left(\frac{\left|s_{j}\right|}{\sqrt{2}}\right)^{2}+\left(\frac{\left|\hat{\delta}_{j}-\hat{\delta}_{j}^{*}\right| \sqrt{\psi_{j}}}{\sqrt{2}}\right)^{2}\right)^{\frac{1}{2}} \\
& \leq-\min \left(\sqrt{2} \Delta_{1 j}, \frac{\sqrt{2} \Delta_{2 j}}{\sqrt{\psi_{j}}}\right) \sqrt{V_{j}^{*}}
\end{aligned}
$$

According to the finite time stability theorem in [30], the Lyapunov function can converge to zero in finite time under the proposed controller with the adaptive law, also the sliding mode surface can reach zero in finite time, and the convergence time satisfies:

$$
t_{s j} \leq \frac{2}{\min \left(\sqrt{2} \Delta_{1 j}, \frac{\sqrt{2} \Delta_{2 j}}{\sqrt{\psi_{j}}}\right)}\left(V_{j}^{*}(0)\right)^{\frac{1}{2}}, \quad j=\alpha, l
$$

\section{References}

[1] Bandyopadhyay, S., Foust, R., Subramanian, G.P., Chung, S.J., Hadaegh, F.Y., Review of formation flying and constellation missions using nanosatellites. J. Spacecraft Rockets. 53(3), 567-578 (2016). 
[2] Huang, P., Zhang, F., Chen, L., Meng, Z., Zhang, Y., Liu, Z., Hu, Y., A review of space tether in new applications. Nonlinear Dynam. 94(1), 1-19 (2018).

[3] Shi, G., Zhu, Z., Zhu, Z H., Dynamics and control of tethered multi-satellites in elliptic orbits. Aerosp. Sci. Technol. 91, 41-48 (2019).

[4] Misra, A K., Pizzaro-Chong, A., Dynamics of tethered satellites in a hub-spoke formation. Adv. Astronaut. Sci. 117, 219-229 (2004).

[5] Yu, B S., Huang, Z., Geng, L., Jin, D P., Stability and ground experiments of a spinning triangular tethered satellite formation on a low earth orbit. Aerosp. Sci. Technol. 92, 595-604 (2019).

[6] Qi, R., Shi, A., Misra, A K., Kumar, K D., Zhang, J., Coulomb tether double-pyramid formation a potential configuration for geostationary satellite collocation. Aerosp. Sci. Technol. 84, 1131-1140 (2019).

[7] Li, G., Zhu, Z H., Du, C., Meguid, S A., Characteristics of coupled orbital-attitude dynamics of flexible electric solar wind sail. Acta Astronaut. 159, 593-608 (2019).

[8] Huang, P., Hu, Y., Zhao, Y., Ma, J., Zhang, F., Meng, Z., Liu, Z., Coordinated formation control strategy of the rotating hub-spoke tethered formation system. P. I. Mech. Eng. G-J. Aer. 232(2), 317-330 (2018).

[9] Zhao, J., Cai, Z., Nonlinear dynamics and simulation of multi-tethered satellite formations in Halo orbits. Acta Astronaut. 63, 673-681 (2008).

[10] Avanzini, G., Fedi, M., Refined dynamical analysis of multi-tethered satellite formations. Acta Astronaut. 84, 36-48 (2013).

[11] Avanzini, G., Fedi, M., Effects of eccentricity of the reference orbit on multi-tethered satellite formations. Acta Astronaut. 94, 338-350 (2014).

[12] Yudintsev, V., Aslanov, V., Detumbling space debris using modified yo-yo mechanism. J. Guid. Control Dyn. 40(3), 714-721 (2017).

[13] Williams, P., Optimal deployment/retrieval of a tethered formation spinning in the orbital plane. J. Spacecraft Rockets. 43(3), 638-650 (2006).

[14]Kang, J., Zhu, Z H., Wang, W., Wang, C., Li. A., Dynamics and de-spin control of massive target by single tethered space tug. Chinese J. Aeronaut. 32(3), 653-659 (2019).

[15]Wong, B., Misra, A., Planar dynamics of variable length multi-tethered spacecraft near collinear Lagrangian points. Acta Astronaut. 63, 1178-1187 (2008).

[16]Zhao, J., Cai, Z., Qi, Z., Dynamics of variable-length tethered formations near libration points. J. Guid. Control Dyn. 33(4), 1172-1183 (2010).

[17]Luo, C Q., Sun, J L., Wen, H., Jin, D P., Dynamics of a tethered satellite formation for space exploration modeled via ANCF. Acta Astronaut. 177, 882-890 (2020).

[18]Pizarro-Chong, A., Misra, A K., Dynamics of multi-tethered satellite formations containing a parent body. Acta Astronaut. 63, 1188-1202 (2008).

[19]Zhai, G., Su, F., Zhang, J., Liang, B., Deployment strategies for planar multi-tethered satellite formation. Aerosp. Sci. Technol. 71, 475-484 (2017). 
[20]Huang, P., Zhao, Y., Zhang, F., Ma, J., Meng, Z., Liu, Z., Zhang, Y., Deployment/retraction of the rotating hub-spoke tethered formation system. Aerosp. Sci. Technol. 69, 495-503 (2017).

[21] Guang, Z., Xingzi, B., Bin, L., Optimal deployment of spin-stabilized tethered formations with continuous thrusters. Nonlinear Dynam. 95(3), 2143-2162 (2019).

[22]Chen, S., Li, A., Wang, C., Liu, C., Adaptive sliding mode control for deployment of electrodynamic tether via limited tension and current. Acta Astronaut. 177, 842-852 (2019).

[23]Liu, C., Wang, W., Guo, Y., Chen, S., Li, A., Wang C., Neural network-based adaptive terminal sliding mode control for the deployment process of the dual-body tethered satellite system. P. I. Mech. Eng. G-J. Aer. 234(6), 1157-1171 (2019).

[24]Kang, J., Zhu, Z H., Wang, W., Li, A., Wang, C., Fractional order sliding mode control for tethered satellite deployment with disturbance. Adv. Space Res. 59(1), 263-273 (2017).

[25] Xu, S., Sun, G., Ma, Z., Li, X., Fractional-order fuzzy sliding mode control for the deployment of tethered satellite system under input saturation. IEEE T. Aero. Elec. Sys. 55(2), 747-756 (2018).

[26] Ma, Z., Sun, G., Adaptive hierarchical sliding mode control with input saturation for attitude regulation of multi-satellite tethered system. J. of Astronaut. Sci. 64(2), 207-230 (2017).

[27]Hallaj, M., Assadian, N., Sliding mode control of electromagnetic tethered satellite formation. Adv. Space Res. 58(4), 619-634 (2016).

[28] Xu, R., Özgüner, Ü., Sliding mode control of a class of underactuated systems. Automatica. 44(1), 233-241 (2008).

[29] Yang, L., Yang. J., Nonsingular fast terminal sliding-mode control for nonlinear dynamical systems. Int. J. Robust Nonlin. 21(16), 1865-1879 (2011).

[30]Bhat, S P., Bernstein, D S., Finite-time stability of continuous autonomous systems. SIAM J. Control Optim. 38(3), 751-766 (2000).

[31]Beukers, F., Gauss' hypergeometric function Arithmetic and geometry around hypergeometric functions. Prog. Math. 260, 23-42 (2007). 